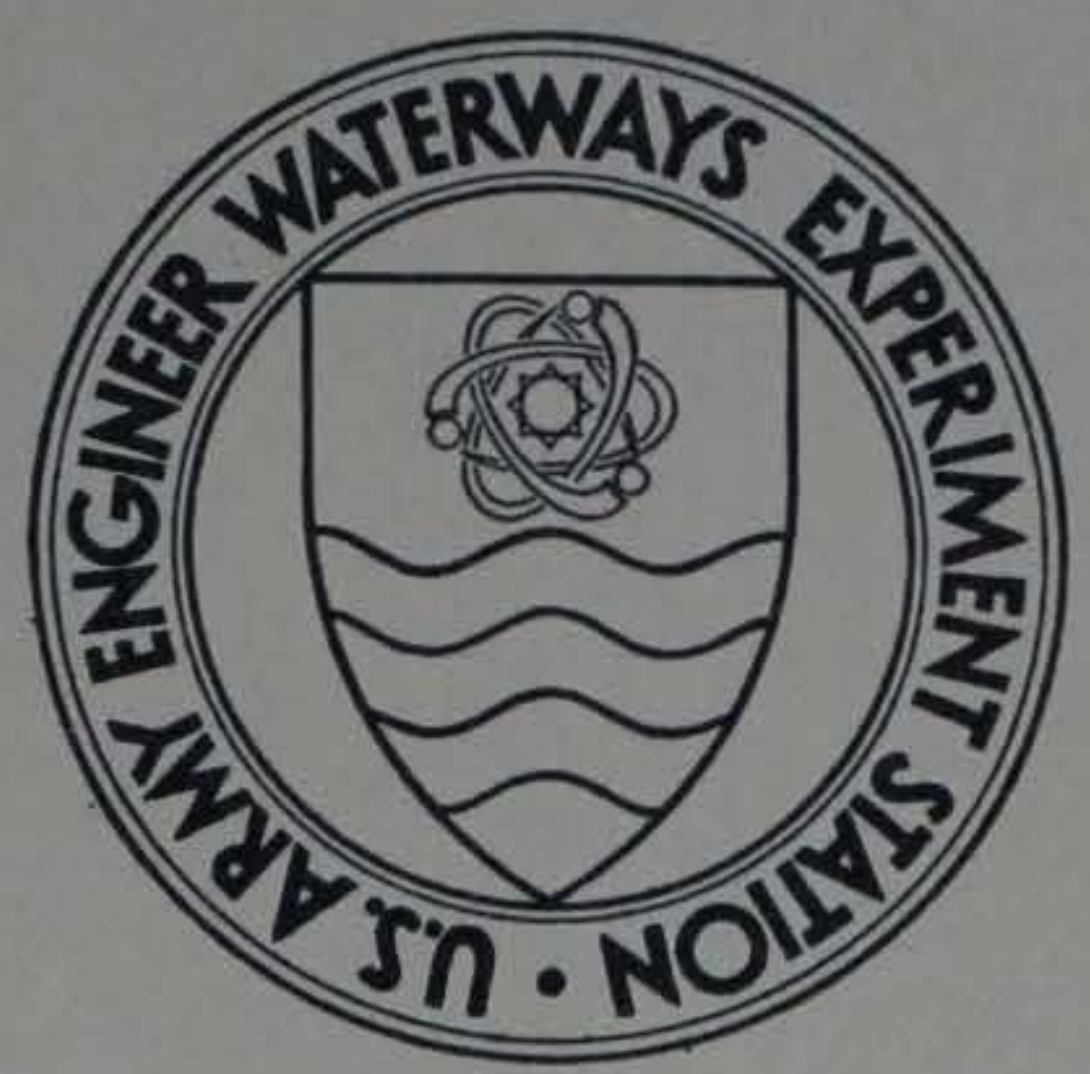

MISCELLANEOUS PAPER N-70-I

\title{
DIGITAL FILTERS FOR ROUTINE DATA REDUCTION
}

by

H. D. Carleton

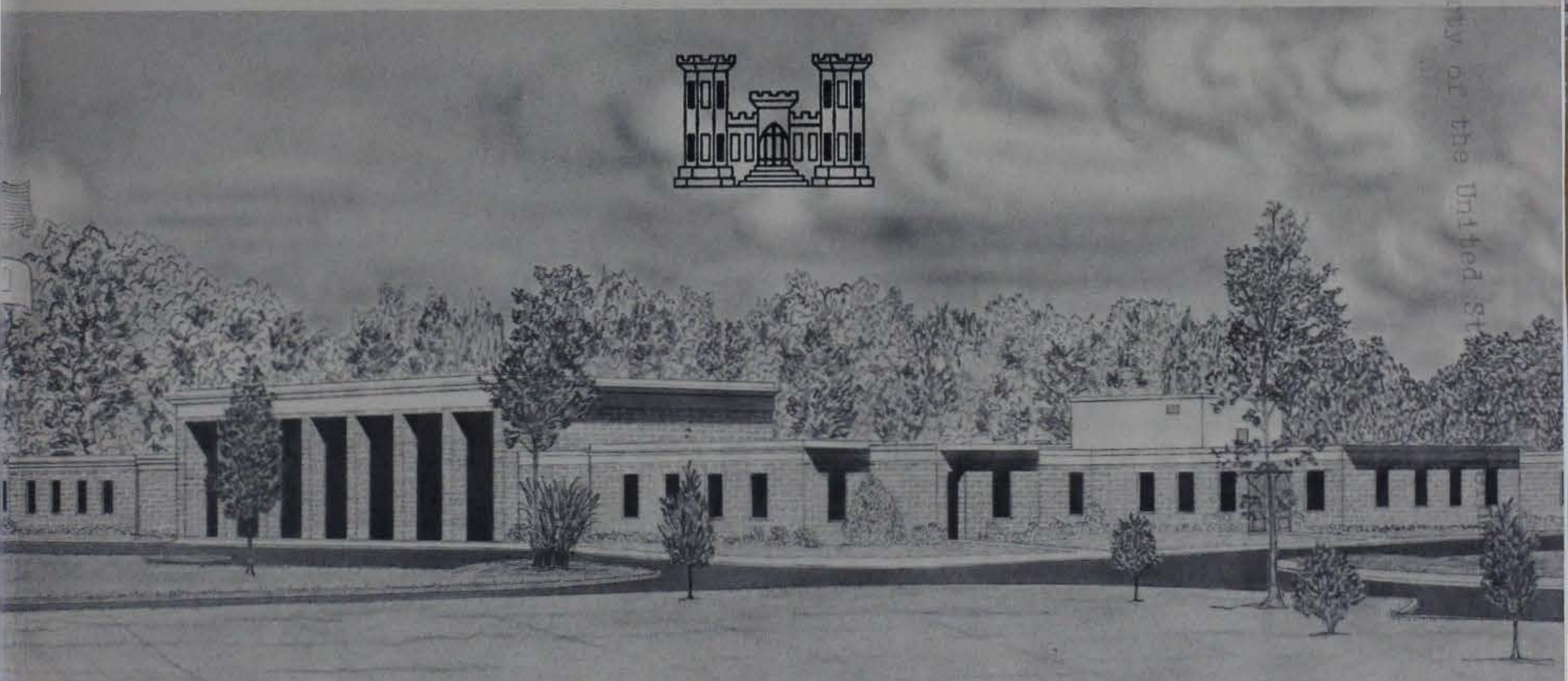

March 1970

RESEARCH CENTER LIBRARY

US RRMY EKGINEER WATERWAYS EXPERIMENT STATIOH

VICKSBURG, MISSISSIPPI

Published by U. S. Army Engineer Waterways Experiment Station, Vicksburg, Mississippi 


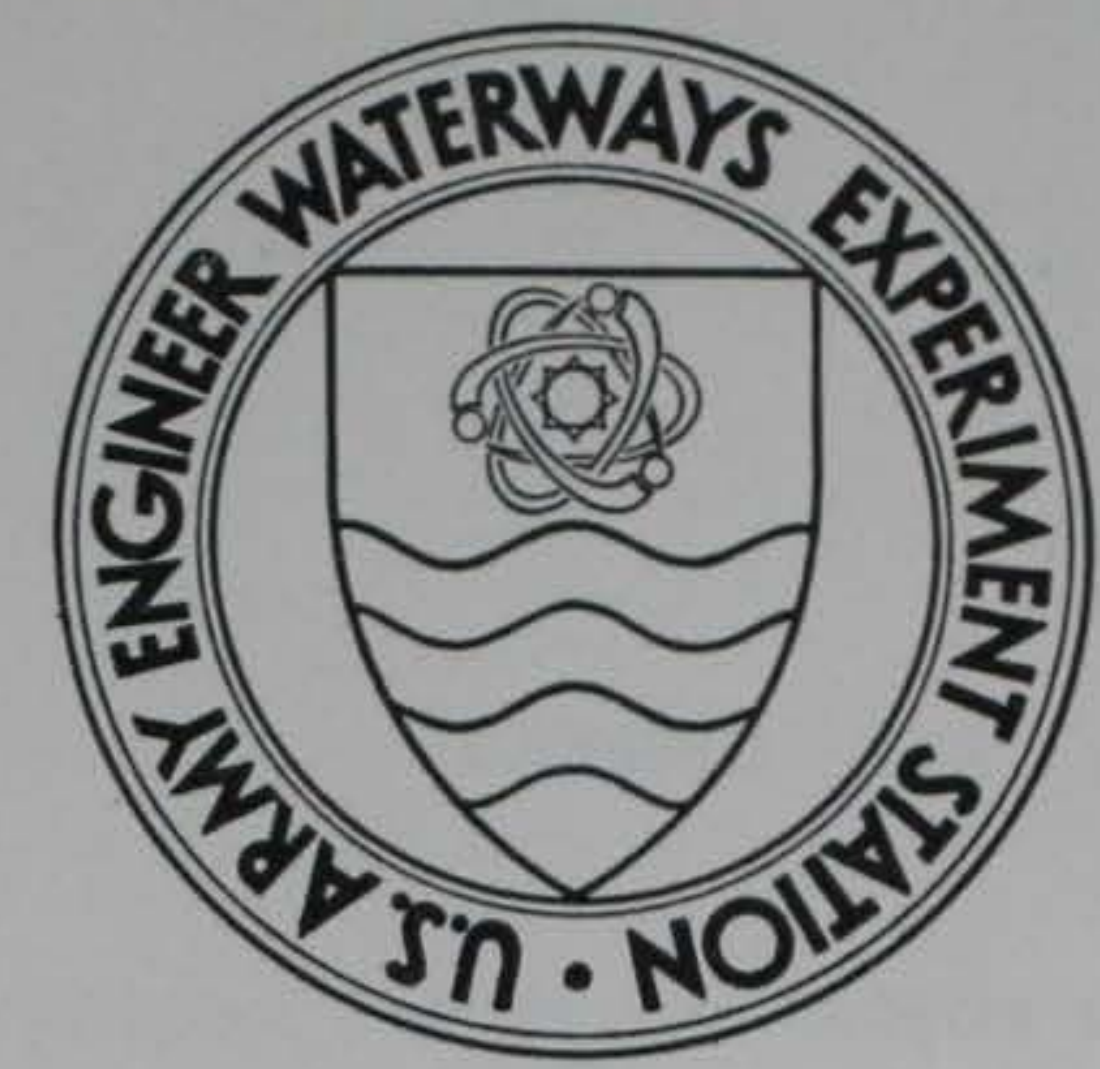

MISCELLANEOUS PAPER N-70-I

\title{
DIGITAL FILTERS FOR ROUTINE DATA REDUCTION
}

\author{
by \\ H. D. Carleton
}

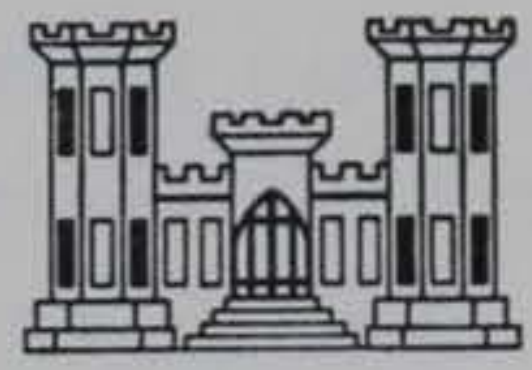

March 1970

Published by U. S. Army Engineer Waterways Experiment Station, Vicksburg, Mississippi 
FOREWORD

This paper was prepared for presentation at the Air Force Special Weapons Center Symposium, "Instrumentation for Nuclear Weapons Effects Simulation," 12-13 March 1970.

The filter set described in this paper was developed during normal operation of the Physical Sciences Branch, Nuclear Weapons Effects Division, U. S. Army Engineer Waterways Experiment Station (WES), under the general supervision of Mr. G. L. Arbuthnot, Jr., Chief, Nuclear Weapons Effects Division, and Mr. L. F. Ingram, Chief, Physical Sciences Branch. $\mathrm{Mr}$. H. D. Carleton was responsible for project development and documentation. The filters of the set were incorporated into computer program number 803-G9RO-118 during the period from June to December 1969 by Mr. J. T. Brogan, assisted by Mrs. C. J. Pittman.

This work was performed to improve the quality of ground shock data obtained on field tests sponsored largely by the Defense Atomic Support Agency (DASA). Additional funding support was provided from DASA Project 61102H-L11CAXSX502, "Ground Stress and Motion Measurements." Computer programs developed as a result of this work are also being used for the processing of laboratory test data obtained from various nuclear weapons effects research (NWER) studies in the WES blast load generator facility. WES Director during the filter set's development was COL Levi A. Brown. Technical Director was Mr. F. R. Brown. 
CONTENTS

FOREWORD . . . . . . . . . . . . . . . . . . . . . . .

$\underline{\text { Page }}$

NOTATION . . . . . . . . . . . . . . . . . . . . . . . . . .

SUMMARY .

PART I: DIGITAL DATA SAMPLING . . . . . . . . . . . . . . . .

Introduction . . . . . . . . . . . . . . . . . . . . . . .

Notes on Discrete Sampling

Sampling Frequency Selection

PART II: THE RANDOM-SPIKE REJECTION FILTER. . . . . . . . . . . .

PART III: SELECTIVE FREQUENCY REJECTION . . . . . . . . . . . . .

Complex Plane Frequency Representations. . . . . . . . . . .

Filter Synthesis . . . . . . . . . . . . . . . . . . . . .

The Zero-Frequency Rejection Filter. . . . . . . . . . . . .

The Single-Frequency Rejection Filter. . . . . . . . . . . .

PART IV: LOW-PASS FILTERS . . . . . . . . . . . . . . . . . . . . 17

Filter Synthesis . . . . . . . . . . . . . . . . 17

The Low-Pass Options . . . . . . . . . . . . . . . . 20

PART V: USE OF THE FILTER SET . . . . . . . . . . . . . . . . . . 25

General. . . . . . . . . . . . . . . . . 25

Random-Spike Rejection. . . . . . . . . . . . . . 25

Low-Pass Operations. . . . . . . . . . . . . . . . 25

Selective Frequency Rejection. . . . . . . . . . . . . . 26

Exercise of Judgment . . . . . . . . . . . . . . . . 26

LITERATURE CITED . . . . . . . . . . . . . . . . . . . . . . . . . . 28

PLATES $1-10$ 
$a_{u}$ the $u^{\text {th }}$ coefficient in a filter numerator polynomial

$\mathrm{b}_{\mathrm{v}}$ the $\mathrm{v}^{\text {th }}$ coefficient in a filter denominator polynomial

e natural logarithm base

$f \quad$ any desired frequency (hertz)

$f_{A}$ any aliased frequency (hertz)

$f_{N}$ folding or Nyquist frequency (hertz)

$f_{s} \quad$ sampling frequency (hertz)

$j$ the imaginary operator $\sqrt{-I}$

$\mathrm{K}$ maximum power of $\mathrm{z}$ in the numerator of a filter function

I maximum power of $\mathrm{z}$ in the denominator of a filter function

$\mathrm{n}$ sample number (an integer)

s the generalized frequency variable of Laplace

$t$ elapsed time (seconds or sampling units)

T sampling period (seconds)

$x_{n} \quad$ wave displacement value for any considered input time $=$ input sample number $\mathrm{n}$

$\mathrm{x}_{\mathrm{n}+1}$ the input sample immediately following (in time) input sample number $n$

the input sample immediately preceding input sample number $\mathrm{n}$

$x_{n-2}$ the input sample immediately preceding input sample number $\mathrm{n}-1$

$\mathrm{y}_{\mathrm{n}}$ the output sample corresponding to input sample number $\mathrm{n}$ the output sample immediately preceding output sample number $n$ the output sample immediately preceding output sample number $n$ - I

$z$ the $z$ transform variable

$|z|$ the modulus of $z$

$\Delta f \quad$ any difference in frequency (hertz) 
$\eta \quad$ the real component of $\mathrm{z}$

$\nu$ the imaginary component of $\mathrm{z}$

$\sigma \quad$ the real component of $s$

$\omega$ the frequency variable (radians per unit time), and imaginary component of $\mathrm{s}$ 
Digital tapes produced from analog field data often carry over severe noise problems. A batch data processing capability should therefore be complemented by digital filters which can remove noise routinely. This paper describes a set of filters developed for use with Nuclear Weapons Effects Division standard data processing codes. Emphasis in assembly of the set was placed on accuracy of signal retention and on adaptability to general purpose computers.

Part I discusses data which is sampled at equally spaced times, the use of sample numbers to designate time positions, and the requirement for use of a sampling frequency at least eight times greater than the highest expected signal frequency.

Parts II, III, and IV document the filters of the three subroutines presently being used for noise removal. The first subroutine eliminates randomly spaced single-sample "spikes" through the application of inequality conditions to data. The second subroutine provides frequency filters for the removal of zero drift and single noise frequencies. Low-pass frequency filters are available in the third subroutine.

Part $V$ discusses the ten example plates used to demonstrate the filter set's effectiveness and speed of operation. 


\section{PART I: DIGITAL DATA SAMPLING}

\section{Introduction}

1. With the purchase of an off-line analog to digital (A/D) converter in 1968, the Nuclear Weapons Effects Division (NWED) of the U. S. Army Engineer Waterways Experiment Station (WES) obtained a batch data processing capability. Since analog data is often quite noisy, and because the A/D converter changes both signal and noise to digital form, digital techniques for noise removal became an immediate consideration. Studies of several approaches to the noise problem led to the conclusion that a digital filter set should be assembled for routine use. Filters accepted for the set were to cause no phase distortion among passed frequencies and to operate with reasonable economy on the general purpose computer equipment available at WES.

2. This report has been written to document the filter set developed, and to aid project officers in their use of its component filters. These filters are included as subroutines to NWED standard data processing codes. A basic familiarity with complex numbers is assumed for the discussions contained in Parts III and IV.

\section{Notes on Discrete Sampling}

3. If the displacement of a continuous wave form is sampled periodically, the resulting time sequence of equally spaced observations is said to be a "discrete time series." The time represented by any given sample in this series would be $t=n T$, where $n$ is the sample number (counting by units from sample number 0 at $t=0$ ) and $T$ is the sampling period (a constant, commonly in seconds, which is the reciprocal of the sampling frequency $f_{s}$, commonly in hertz). If $T$ is defined as one unit of time, $t=n T$ becomes $t=n$. 
4. Fig. I shows examples of periodically sampled continuous waves. At a given sampling frequency, say for example $6000 \mathrm{~Hz}$, no oscillation can be represented if all sampled displacement values are exactly the same (as in fig. la). This is because actual displacement between samples cannot be determined by examination of the discretely sampled data. Only estimation or interpolation is possible. If all displacement sample values are exactly the same, and not equal to zero, "zero frequency" is represented by the constant offset from the zero displacement base line (displacement equilibrium position).* If only two displacement values exist among all the samples taken, and these two values are found alternating at every change in sample through time (as in fig. Ib), then the frequency represented by this alternation is half the sampling frequency, or for our example, $3000 \mathrm{~Hz}$. This frequency, known as the folding or Nyquist frequency $f_{N}$, is the highest frequency which may be represented by discretely sampled data at a given sampling frequency. Frequencies higher than folding, if they are present on the original continuous data, will be represented by distortion of frequencies below folding. Any frequency $f=f_{N}+$ $\Delta f$, where $0<\Delta f<f_{N}$, will be "seen" by the sampling process as the "folded" or "aliased" frequency, $f_{A}=f_{N}-\Delta f$. For the example sample rate of $6000 \mathrm{~Hz}$, for instance, $4200-\mathrm{Hz}$ noise, if present, would be "frequency aliased" as $1800 \mathrm{~Hz}$ and added to any existing 1800-Hz signal, since folding frequency would be $3000 \mathrm{~Hz}$. (Additional discussion of aliasing may be found in Blackman and Tukey, 1 pp 216-219 and 521-524.)

5. It has been stated in the preceding paragraph that folding frequency is the highest frequency which may be represented by discretely sampled data at a given sampling frequency. A reasonably accurate approximation of folding frequency sine waves can be made only where the times of sampling correspond to the times of maximum displacement for a wave (as in fig. Ib). If the times of maximum wave displacement are out of phase with the sampling times, both displacement and phase angle are unacceptably altered for the wave represented (see fig. 2a). In fact, a 90-deg phase difference between sample times and maximum displacement times for the folding

* Constants and linear trends are zero frequency. See Blackman and Tukey, ${ }^{1}$ section 19. 


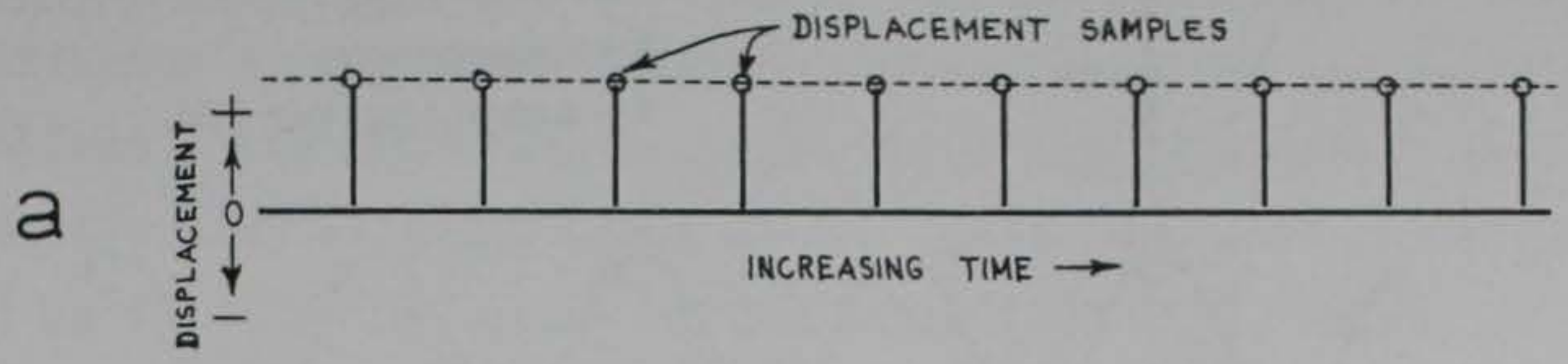

ZERO FREQUENCY - CONSTANT OFFSET

NO OSCILLATION REPRESENTED

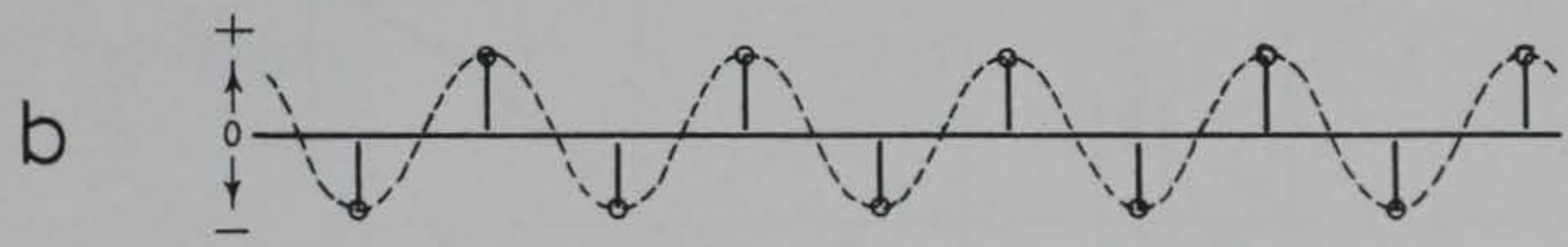

FOLDING FREQUENCY

SINE WAVE REPRESENTED

C
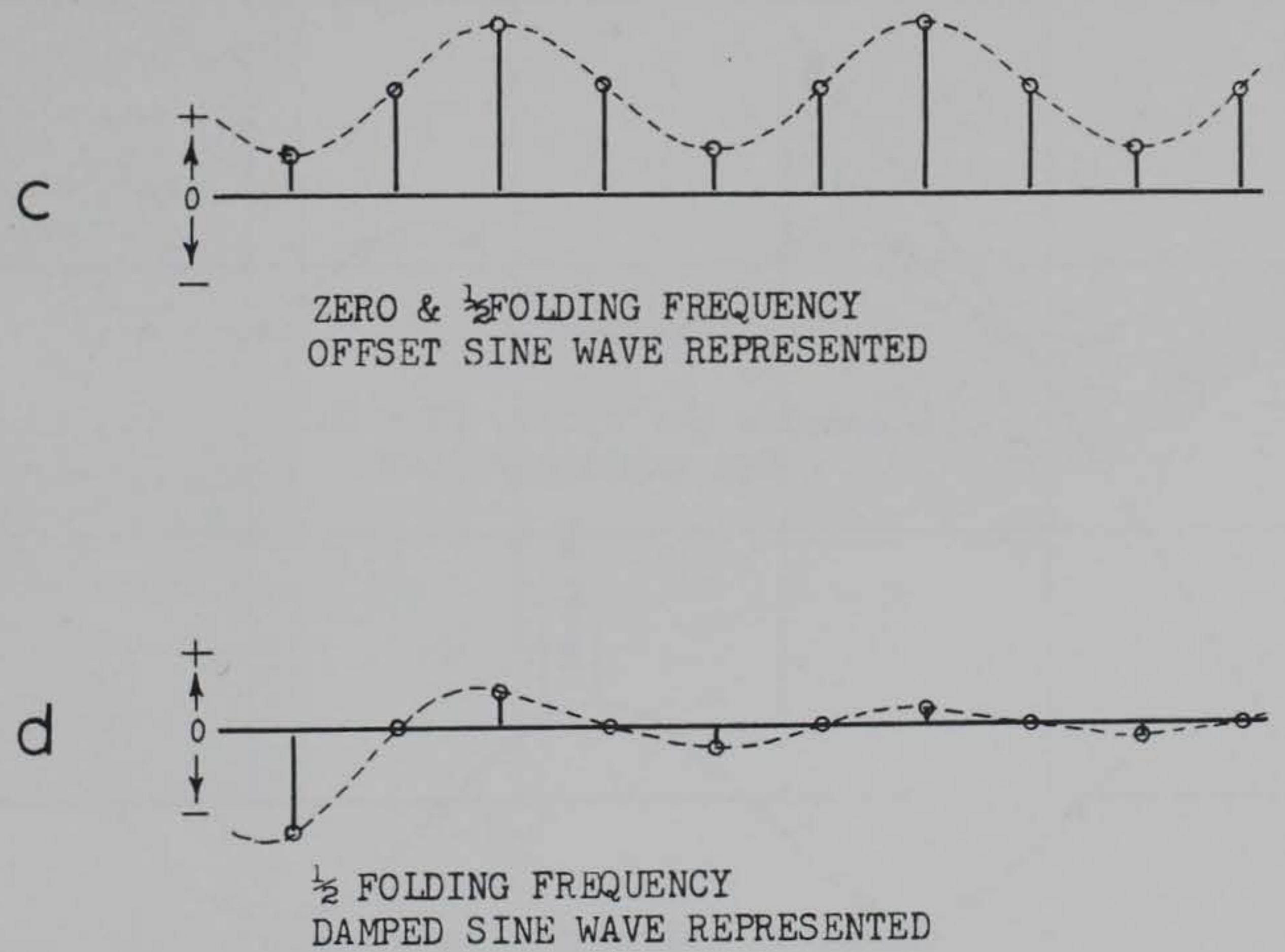

Fig. 1. Periodically sampled continuous waves 

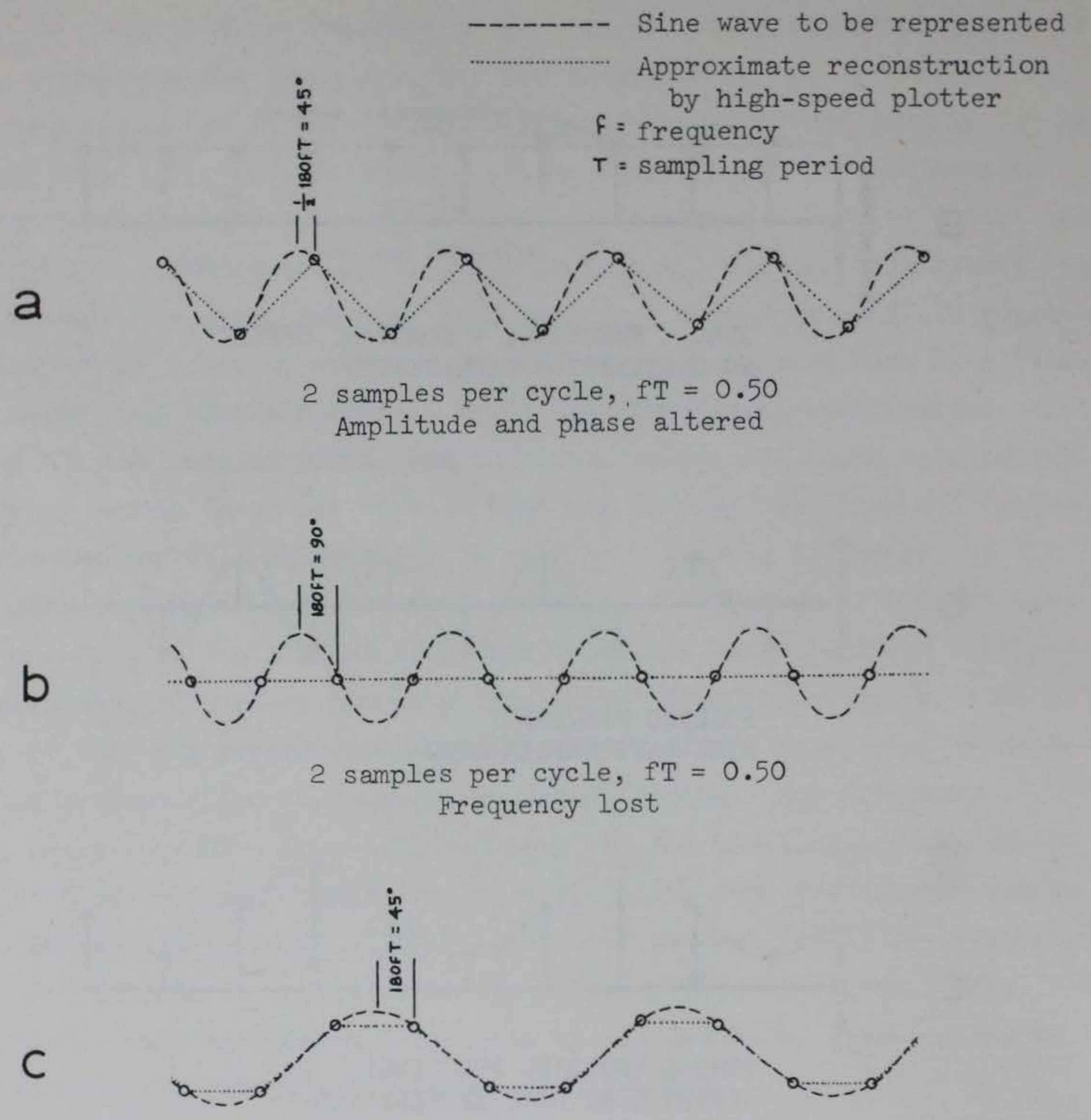

4 samples per cycle, $\mathrm{fT}=0.25$

$29 \%$ amplitude loss

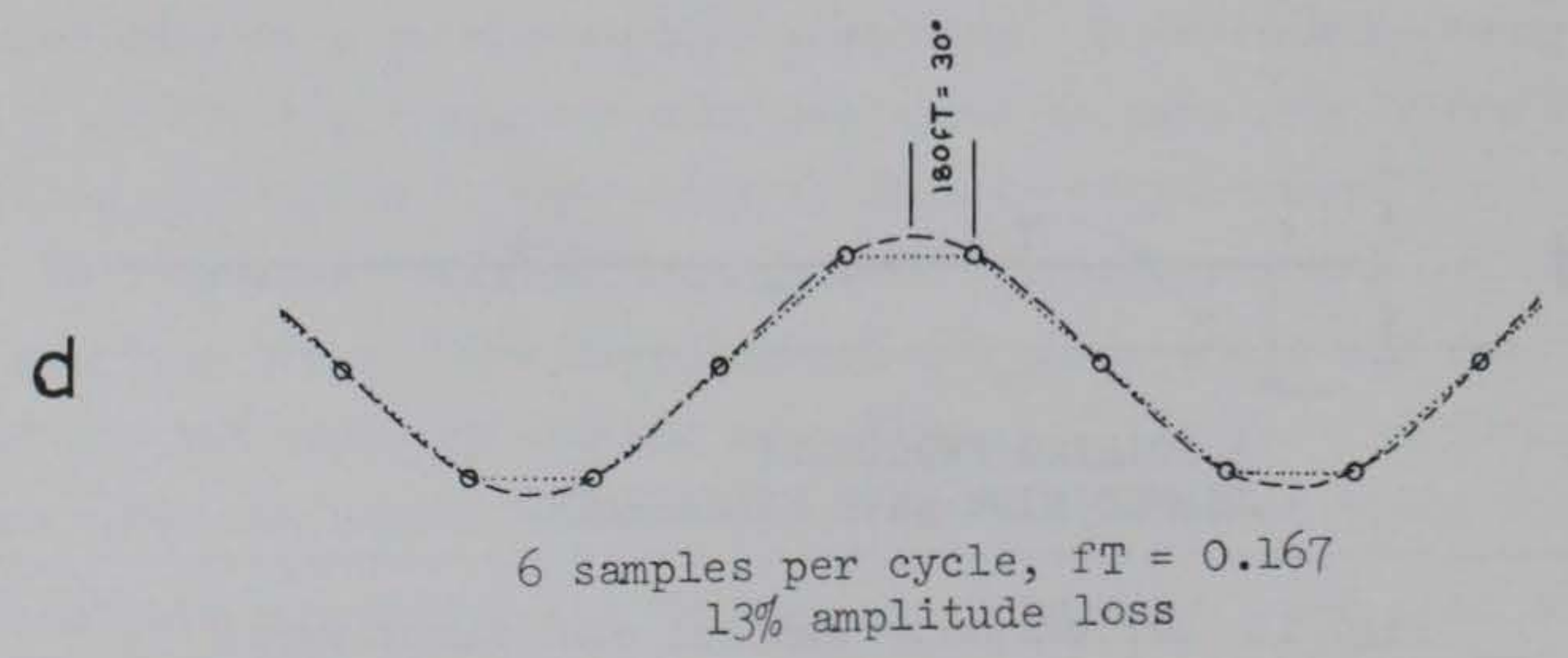

Fig. 2. Distortion of sine waves due to sampling and plotting 
frequency reduces all sample displacements to zero, and no wave is represented (see fig. $2 \mathrm{~b}$ ). Frequencies below, but near, folding are also strongly affected by the phase relation between sampling and maximum displacement times. The amplitude response which may be expected over portions of a wave with sampling times at the least favorable positions may be determined by finding the cosine of the angle $(180 \mathrm{fT})^{\circ}$, where $\mathrm{fT}$ is the reciprocal of the number of samples per cycle for the frequency $f$ being considered (see figs $2 \mathrm{~b}, \mathrm{c}$, and $\mathrm{d}$ ). Fig. 3 is a plot of amplitude responses

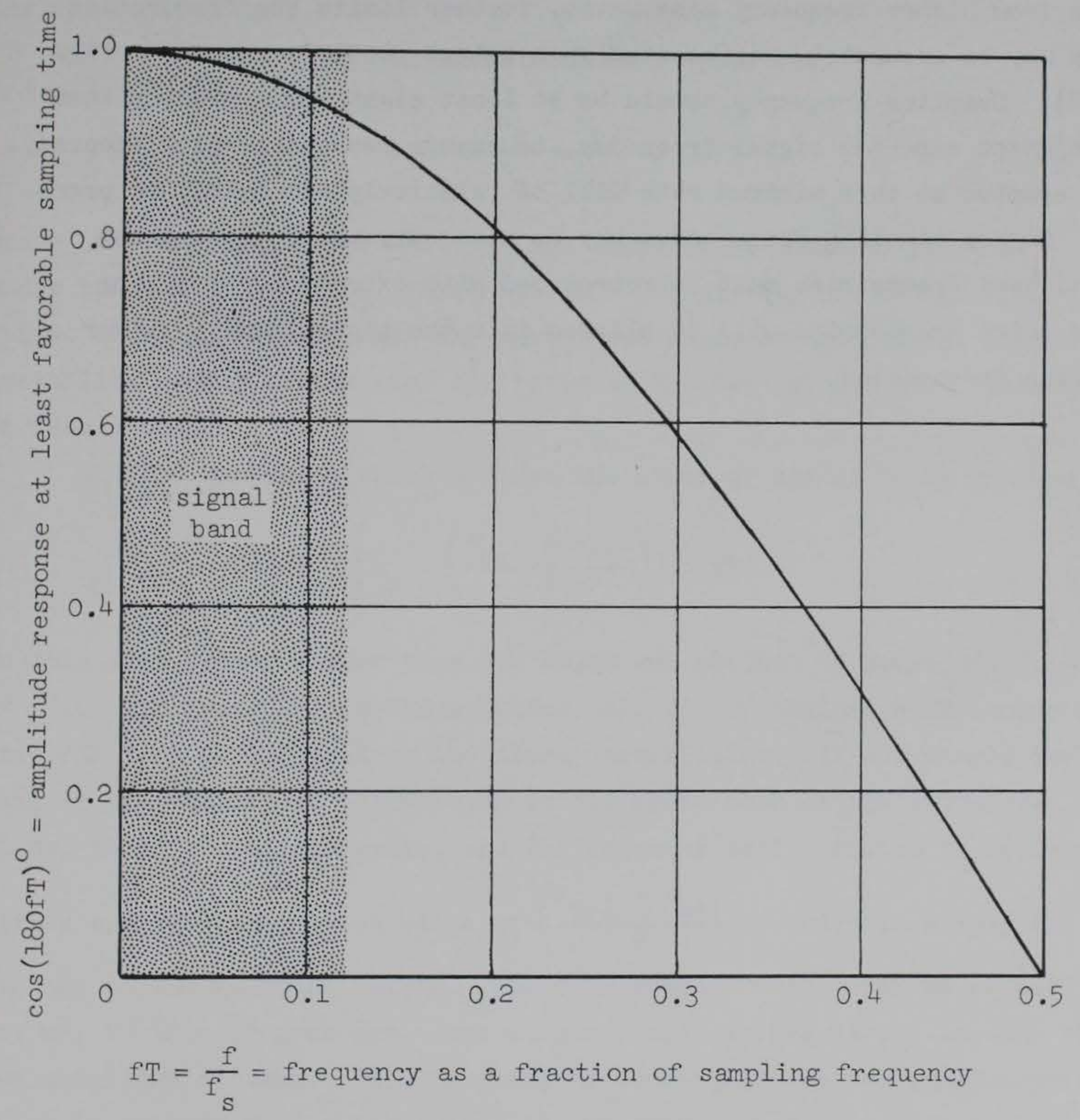

Fig. 3. Minimum apparent amplitude response due to sampling and plotting 
for sampled frequencies at the most unfavorable sampling phase relations.

\section{Sampling Frequency Selection}

6. The selection of a given sampling frequency band limits signal frequency representations by establishing folding frequency at half the frequency of sampling. The plotting process, through its inability to superimpose a sine wave upon all displacement samples which may have been taken from higher frequency sine waves, further limits the frequencies which may be accurately represented upon output plots (as shown in figs. 2 and 3). Sampling frequency should be at least eight times greater than the highest expected signal frequency, to assure reasonable plot accuracy. Data sampled at this minimum rate will be relatively economical to process. Higher sampling frequencies may be necessary where the highest signal band frequencies must be reproduced with extreme accuracy, or where noise frequencies will be aliased into the signal band by lower sampling frequencies. 
7. Digital tapes occasionally have upon them single samples (at apparently random locations in the discrete time series) which show very high displacement values, and which bear no true relation to the seismic data thereon. These spurious "spikes" frequently cause large errors during frequency filtering, integration, and frequency analysis operations. The problem may be eliminated by application of mathematical inequalities to the raw digital data.

8. The main condition which defines a spike is:

$$
1.25>\frac{x_{n}-x_{n-1}}{x_{n}-x_{n+1}}>0.75
$$

where $x_{n}$ represents the value of the input (displacement) presently under consideration, $x_{n-1}$ represents the immediately preceding input value, and $x_{n+1}$ represents the next input value in the future. The above inequality simply states that the ratio of the two "sides" of a spike must be nearly unity.

9. The condition which defines the sizes of spikes to be removed is:

$$
\left|x_{n}-\left(\frac{x_{n-1}+x_{n+1}}{2}\right)\right|>220
$$

In this case spikes larger than 220 units are defined as spurious.

10. Instructions to the computer tell it to consider each sample in a . discrete time series against the above inequalities. If any sample (relative to the samples on either side of it) meets both of the above conditions, it is a spurious spike, and the computer is instructed to replace it with a sample displacement value of $\left(\frac{x_{n-1}+x_{n+1}}{2}\right)$, which is simply the average of the adjacent values. The only samples which will be affected in any way by this program are those single sample spikes larger in size than the specified minimum. This minimum, of course, may be carefully altered where it appears from outputs that the program is not operating at best efficiency. 


\section{PART III: SELECTIVE FREQUENCY REJECTION}

\section{Complex Plane Frequency Representations}

11. The discussion in this section relates graphical frequency representations to Fourier series theory and Laplace's generalization of this theory. (See a text such as LePage ${ }^{2}$ for background. Additional information may be found in Blackman and Tukey, ${ }^{1}$ pp 252-258.)

12. The Fourier frequency variable $\omega$ (expressed in radians per unit time) is related to Laplace's $s$, a generalization of $\omega$, as follows: $e^{S T}$ is a generalization of $e^{j \omega T}$ (where $T$ is the sampling period, a constant), and $s=\sigma+j \omega$, where:

\section{$s$ is called the "generalized frequency variable" \\ $\sigma$ is the real component of $s$ \\ $j$ is the imaginary operator $\sqrt{-1}$ \\ $\omega$ is the frequency variable, and the imaginary component of $\mathrm{s}$}

Therefore, $s$ is a complex number, the value of which is represented by the ordered pair of real numbers $(\sigma, \omega)$ (see fig. 4 ). While itself real, $\omega$ is used in this terminology as the multiplier of $j$ in the $s$ plane. When $\sigma=0, s=j \omega$, and $e^{S T}=e^{j \omega T}=z$. In this specific case, values of

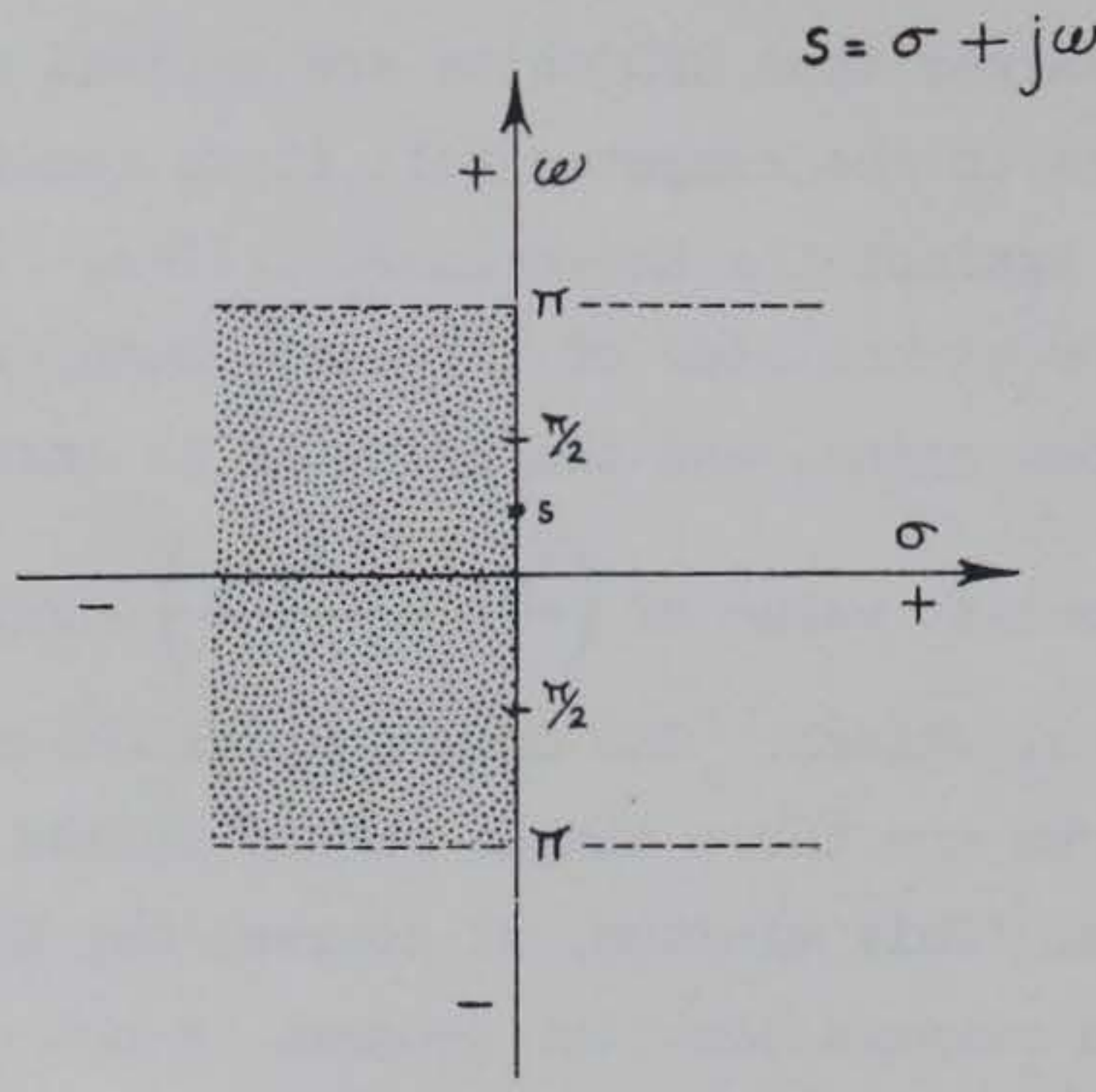

Fig. 4. s plane representation of frequency 
$z$ vary as $\omega$ changes, since $T$ is a constant.

13. The $s$ plane is used to define values of $s$, the generalized frequency variable. If the function $e^{s T}$ of the independent complex variable $s$ is plotted on a second complex plane (the $z$ plane) as $s$ follows a path along the $s$ plane imaginary axis from $(0,-\pi)$ to $(0, \pi)$, the unit circle (modulus, $|z|=1$ ) will result (see fig. 5). This mapping is done through the use of Euler's identity, $e^{j \omega T}=\cos \omega T+j \sin \omega T$, where wI is the phase angle on the $z$ plane. This angle represents

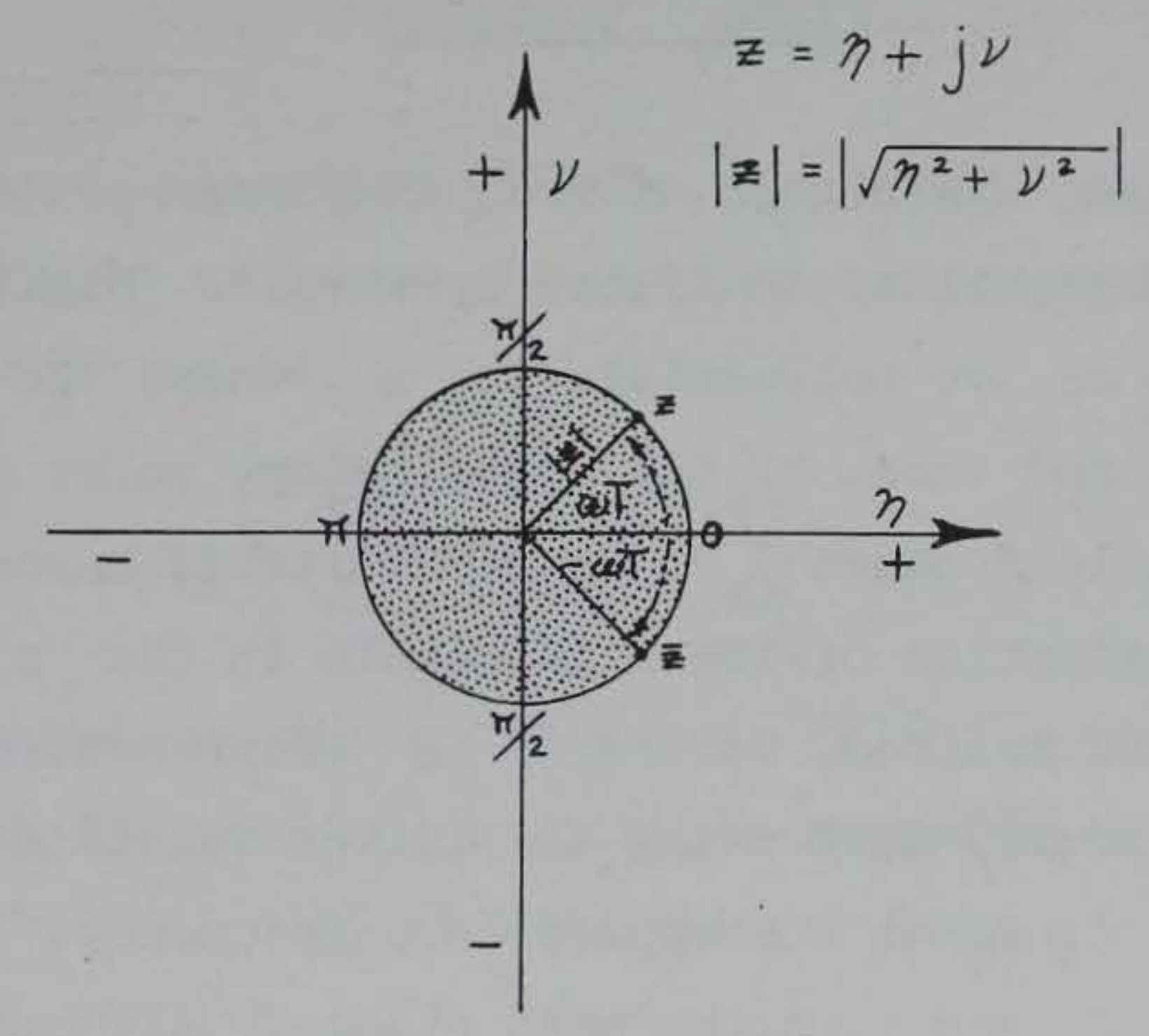

Fig. 5. z plane representation of frequency

that portion of a full cycle or period at any frequency $f$ which would be completed during one sampling period $T$. Half the unit circle ( $\pi$ radians), then, represents folding frequency $f_{\mathbb{N}}$ since folding is half of the sampling frequency, and only half of a cycle would be completed during one sampling period. In terms of folding frequency:

$$
\omega T=2 \pi f T=2 \pi f \frac{1}{2 f_{N}}=\frac{\pi f}{f_{N}}
$$

Zero frequency is represented by $z=(1,0)$, and folding frequency by $z=(-1,0)$. Values of frequency between zero and folding are represented by $\omega T$ intercepts between $O$ and $\pi$ on both the upper and lower halves of 
the unit circle, reflecting the band limited nature of frequency spectra for discrete time series. Complex conjugate values of $z$ (conjugate values from above and below the real axis) are used to produce real coefficients in the synthesis of filter polynomials. If $T$ is taken as unity, $\omega$ becomes equivalent to the phase angle on the $z$ plane. (Additional discussion of frequency representations may be found in Robinson and Treitel. ${ }^{3}$ )

\section{Filter Synthesis}

14. An important usefulness of frequency representation in the $\mathrm{z}$ plane lies in its adaptability to filter synthesis. Digital filters may be expressed as a ratio of two polynomials in $z$, where the roots of the numerator are zeros, and those of the denominator, poles (Shanks, ${ }^{4}$ pp 3541). For simple single frequency rejection filters, zeros and poles may be determined by examination of the unit circle in the $z$ plane. Because frequency rejection is desired, values of $\omega$ (represented by $z$ plane points on the unit circle) must cause the filter function (the ratio of two polynomials) to go to zero at the appropriate frequency. This is done by the choice of a zero or zeros on the unit circle. All other values of $w$, however, must produce a filter function response as near as possible to unity, since distortion of nonrejected frequencies is not desirable. The selection of a pole or poles just outside of the unit circle at the same phase angle(s) as the zero(s) will produce this result, at the same time keeping the function stable. (Because $z$ plane representations of $\omega$ (where $\sigma=0$ ) are confined to the unit circle, the filter function will not become infinite at any considered point.)

\section{The Zero-Frequency Rejection Filter}

15. An offset oscillating time function can be made to seek the zero displacement base line as its equilibrium position by application of a zero-frequency digital filter designed by the method outlined in the preceding paragraph. As shown previously, zero frequency is represented by 
the point $z=(1,0)$ on the unit circle. The linear polynomial having $1+j 0$ as its root is $1-z$, which will be the numerator of the zerofrequency rejection filter function. Choosing a pole value on the $\omega=0$ radial but just outside of the unit circle, we have $z=(1.01,0)$. The linear polynomial having $1.01+j 0$ as its root is $1.01-z$, the denominator of the zero-frequency reject filter function. The filter function, then, is:

$$
F(z)=\frac{1-z}{1.01-z}
$$

16. A recursive algorithm (Shanks, ${ }^{4} \mathrm{pp}$ 34-35) may be used to apply this filter function to an input time series. The general recursion equation for rational filters is:

$$
y_{n}=\sum_{u=0}^{K} a_{u} x_{n-u}-\sum_{v=1}^{L} b_{v} y_{n-v}
$$

Where a values represent the coefficients in the numerator polynomial, b values represent the coefficients in the denominator polynomial, $x$ values represent input displacement samples, and $y$ values represent output displacement samples. The values of $u$ and $v$ are taken from the powers of $z$ associated with the coefficients of the filter function polynomials, and $\mathrm{n}$ is the sample number of the output displacement sample being computed. $K$ is the maximum power of $z$ represented in the numerator, and $I$ the maximum power of $z$ found in the denominator. The coefficient of the first term in the denominator $b_{0}$ should be unity. Where this is not the case for a particular filter function, both numerator and denominator are divided by the initial value of $b_{0}$ (excepting the case where $b_{0}=0$ ) to bring $b_{0}$ to unity.

17. In the case of the zero-frequency rejection filter function, numerator and denominator must be divided by 1.01 , which gives:

$$
F(z)=\frac{0.990099-0.990099 z}{1-0.990099 z}
$$

By application of the recursion summation (equation 3): 


$$
\begin{gathered}
y_{n}=0.990099 x_{n}-0.990099 x_{n-1}+0.990099 y_{n-1} \\
y_{n}=0.990099\left(x_{n}-x_{n-1}+y_{n-1}\right)
\end{gathered}
$$

Equation 5 may be used directly in an appropriate computer program to remove the zero-frequency component from a discrete time series. The recursion method of application for this filter has the advantages of speed and accuracy as compared to other methods of filter application, because the consideration of a previously computed output $\left(\mathrm{y}_{n-1}\right)$ and one previous input value $\left(x_{n-1}\right)$ for each output computation $\left(y_{n}\right)$ eliminates the need for consideration of a very long (theoretically infinite) series of previous inputs for each computed output value. A single operation (called a "pass") with this filter brings an offset wave to the base line exponentially. Thus, early displacement values in the time series are only slightly shifted, and the once-filtered wave may appear to have a "warp" similar to that of a very low frequency harmonic. A second operation, this time on the time reversed output of the first pass, will correct this situation (Shanks, ${ }^{4}$ pp 41-42). The phase response of this "two-pass" filter will be zero for all frequencies. The amplitude response is shown in fig. 6 .

\section{The Single-Frequency Rejection Filter}

18. Where a single frequency has been identified as spurious, it may be removed from a discrete time series by an operation only slightly more involved than that used for the zero-frequency rejection filter. From equation 1 (with the sampling period $T$ taken as unity), it is seen that the angle $\omega$ which represents any temporal frequency $f$ is dependent on the ratio of that frequency to the folding frequency. Recalling that folding frequency is equal to half of the sampling frequency:

$$
\omega=\frac{2 \pi f}{f_{s}}
$$




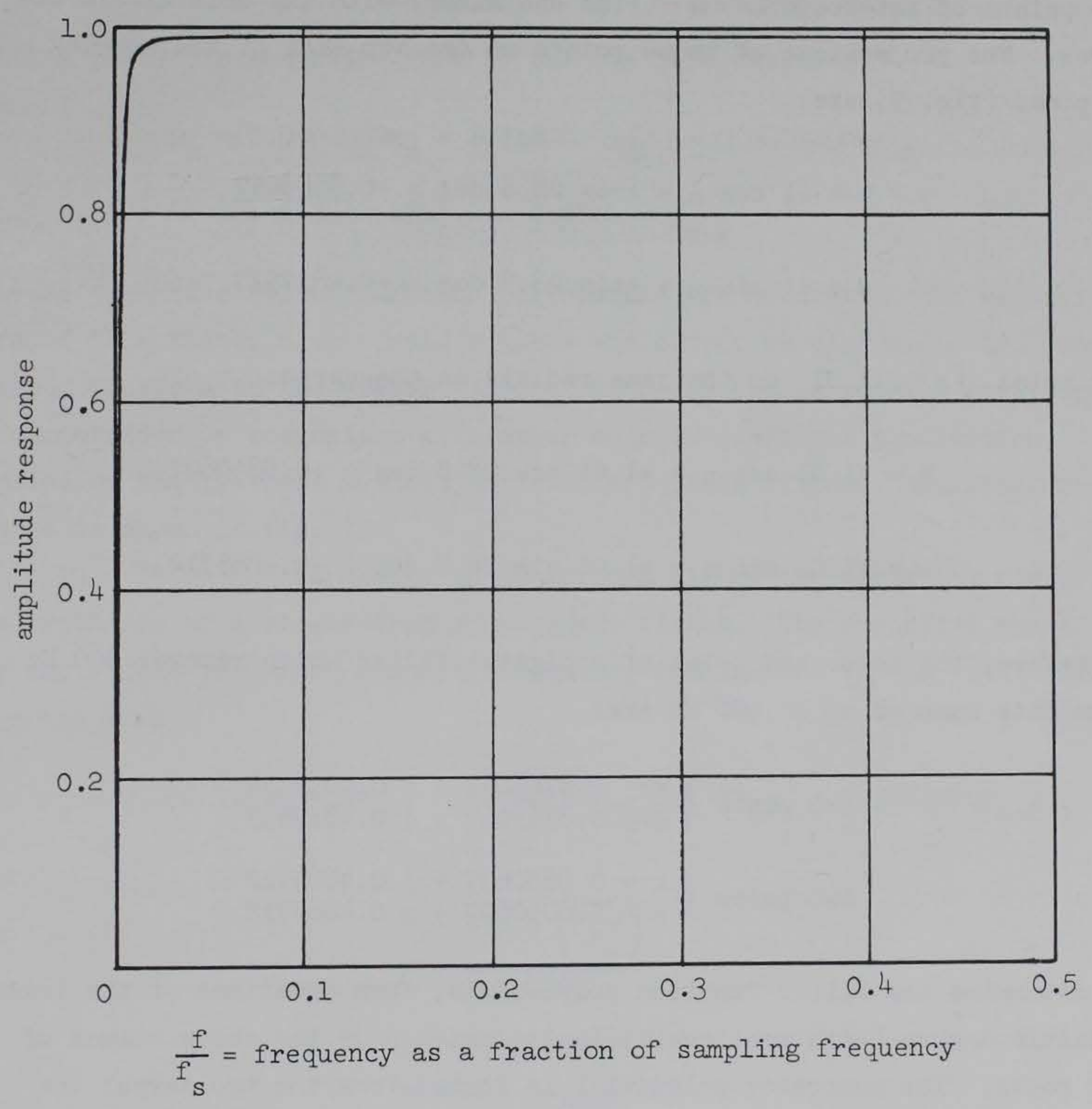

Fig. 6. Amplitude response of zero-frequency rejection filter 
Say that the frequency to be rejected is $960 \mathrm{~Hz}$, * and that sampling frequency is $12,000 \mathrm{~Hz}$.

$$
\omega=\frac{2 \pi(960)}{12,000}=0.5026548 \text { radians }=28.8 \mathrm{deg}
$$

The points of intercept of $\omega$ (plus and minus) with the unit circle are zeros. The projections of these points on the two axes of the complex z plane (fig. 5) are:

$$
\begin{aligned}
& \eta=+1 \cos \omega=+\cos 28.8 \mathrm{deg}=+0.8763067 \\
& \nu= \pm 1 \sin \omega= \pm \sin 28.8 \mathrm{deg}= \pm 0.4817537
\end{aligned}
$$

For poles $|z|=1.01$ on the same radials as the zeros:

$$
\begin{aligned}
& \eta=+1.01 \cos \omega=+1.01 \cos 28.8 \mathrm{deg}=+0.8850697 \\
& \nu= \pm 1.01 \sin \omega= \pm 1.01 \sin 28.8 \mathrm{deg}= \pm 0.4865712
\end{aligned}
$$

Therefore, the zeros and poles of a digital filter which removes $960 \mathrm{~Hz}$ from data sampled at $12,000 \mathrm{~Hz}$ are:

$$
\begin{aligned}
& \text { two zeros }\left\{\begin{array}{l}
z=0.8763067+j 0.4817537 \\
z=0.8763067-j 0.4817537
\end{array}\right. \\
& \text { two poles }\left\{\begin{array}{l}
z=0.8850697+j 0.4865712 \\
z=0.8850697-j 0.4865712
\end{array}\right.
\end{aligned}
$$

To determine the filter function polynomials, form equations of the lowest possible degree (with real coefficients) which have the above values of $z$ for roots. The numerator polynomial is formed from the two zeros; the denominator polynomial from the two poles. For this filter then:

* A common noise frequency--the result of a $60-\mathrm{Hz}$ source and a 16:1 digitizing tape speed ratio, as in plate 9. 


$$
F(z)=\frac{1-1.7526134 z+z^{2}}{1.0201-1.7701395 z+z^{2}}
$$

Dividing both numerator and denominator by 1.0201 to bring $b_{0}=1$ :

$$
F(z)=\frac{0.9802960-1.7180800 z+0.9802960 z^{2}}{1-1.7352608 z+0.9802960 z^{2}}
$$

Putting $F(z)$ in recursive form by the use of equation 3 :

$$
\begin{aligned}
y_{n}= & 0.9802960 x_{n}-1.7180800 x_{n-1}+0.9802960 x_{n-2} \\
& +1.7352608 y_{n-1}-0.9802960 y_{n-2}
\end{aligned}
$$

Because the above filter function polynomials are quadratic, the recursive form of this filter must consider input and output samples up to two sample periods previous to the present input. It nevertheless retains great speed of computation by comparison with other methods of filter application. Applied by the two-pass method, it is a zero phase filter. Amplitude response is shown in fig. 7 .

19. It is not necessary to go through all of the foregoing steps in the synthesis of a single-frequency reject filter. The recursive equation for any reject frequency/sampling frequency combination for $\omega$ will always take the form:

$$
y_{n}=0.9802960 x_{n}-A x_{n-1}+0.9802960 x_{n-2}+B y_{n-1}-0.9802960 y_{n-2}
$$

where

$$
\begin{aligned}
& A=\frac{2 \cos \left(\frac{2 \pi f}{f_{S}}\right)}{1.0201} \\
& B=\frac{2.02 \cos \left(\frac{2 \pi f}{f_{S}}\right)}{1.0201}
\end{aligned}
$$

As an evidence of the exponential nature of this algorithm, recall from Euler's identity that it can be shown that:

$$
\cos \omega=\frac{e^{j \omega}+e^{-j \omega}}{2}
$$


For rejection at $\frac{960 \mathrm{hz}}{12,000 \mathrm{hz}}=0.08$

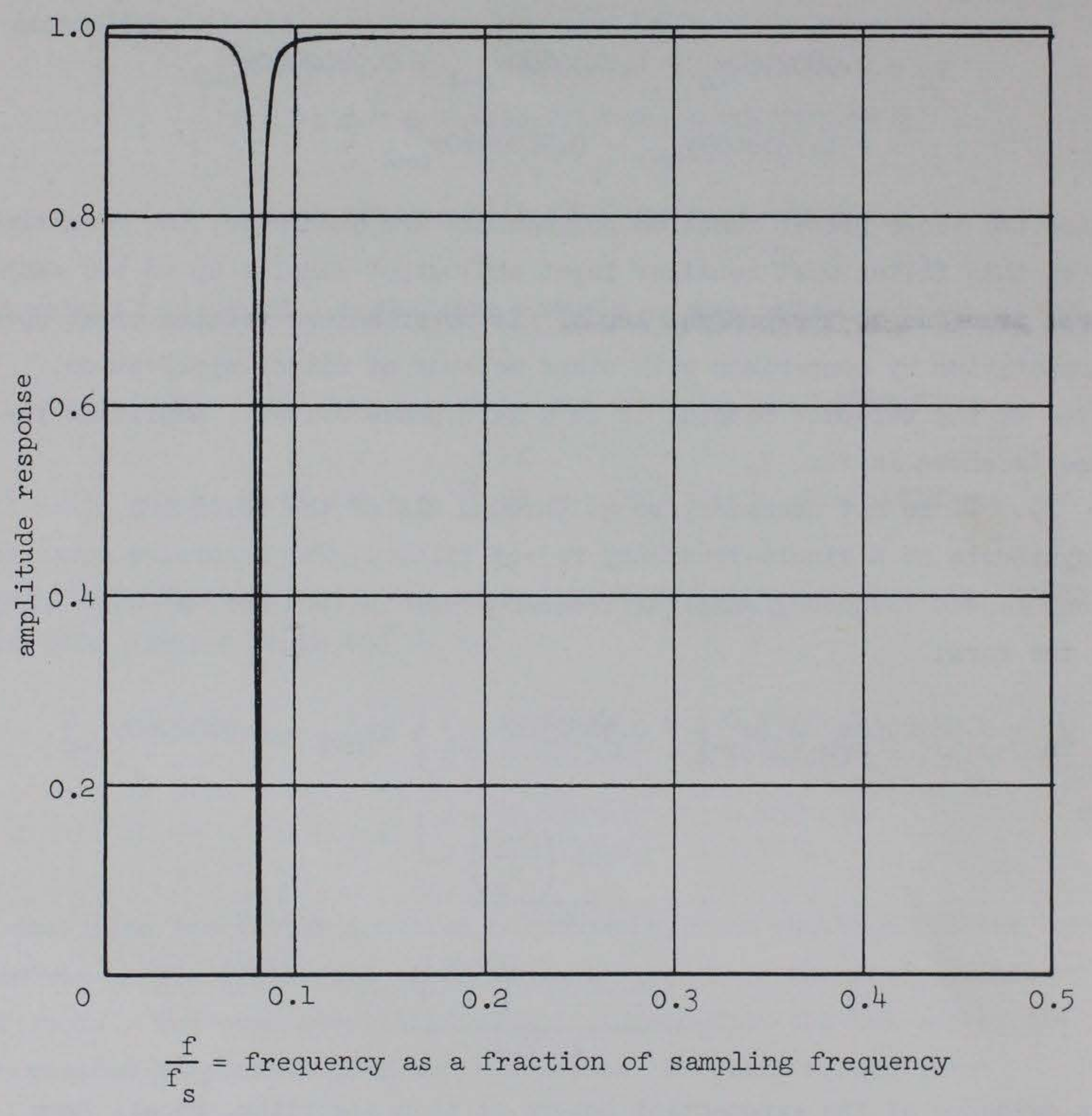

Fig. 7. Amplitude response of single-frequency rejection filter 


\section{Filter Synthesis}

20. Low-pass filters are a virtual necessity where many high noise frequencies accompany shock data. Obviously, a filter function must have numerous zeros if the represented filter's response is to be kept very near zero over a broad band of frequencies. This requirement can lead to filter functions with many terms and coefficients. Whittlesey ${ }^{5}$ suggests an alternative to the use of multicoefficient filter functions. Simple filter functions may be used in series to form composite low-pass filters. This approach limits the choice of low-pass bands to certain specific fractions of the sampling frequency, but filter operation becomes relatively economical, and the routine can be two-passed to eliminate phase distortion.

21. Four composite low-pass filters may be constructed by a proper combination of the following components:

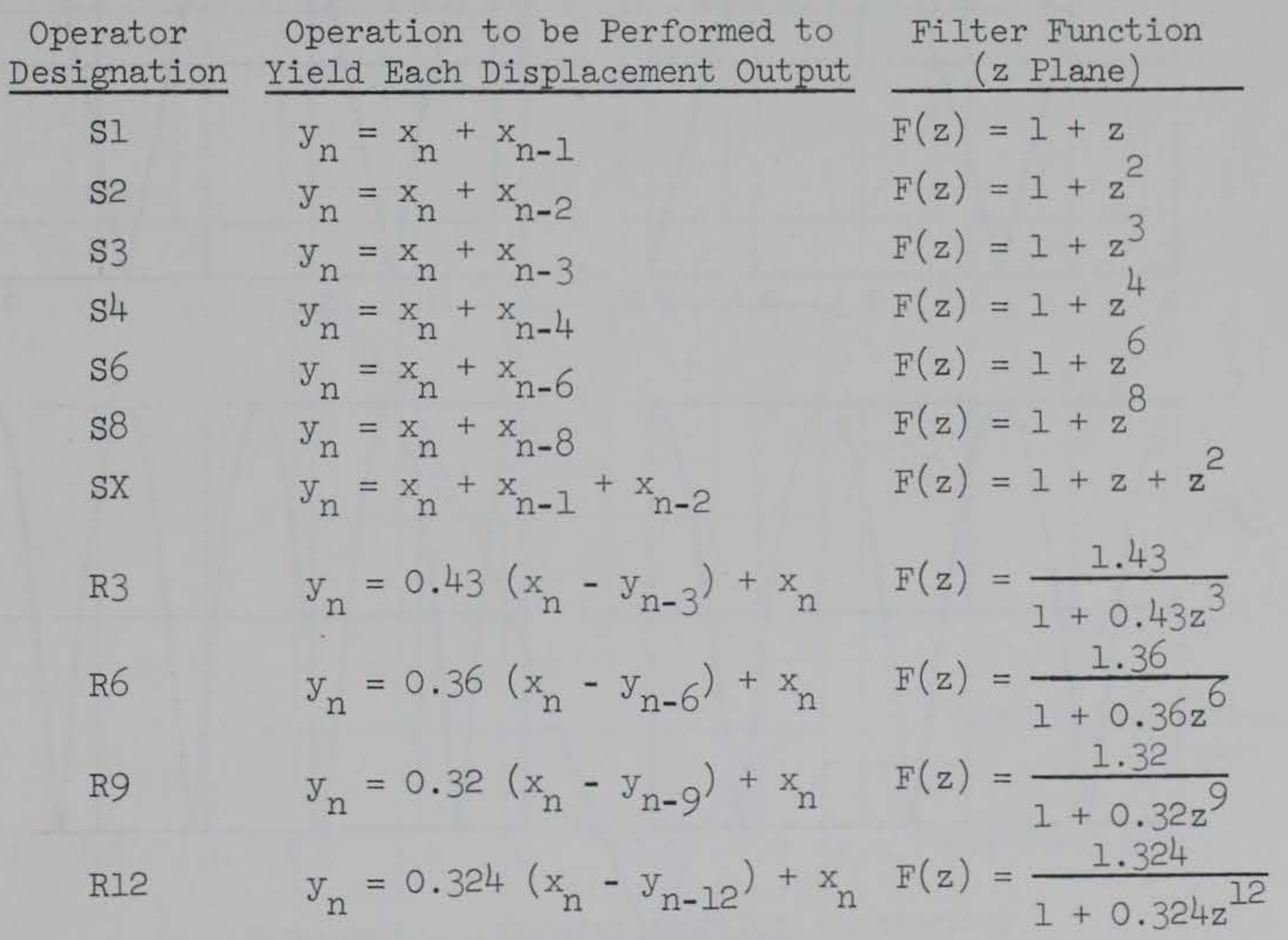

An operator with an "S" designation is a summing operator; one with an 
" $R$ " designation is a recursion operator. Amplitude response plots for these operators are shown in figs. 8 and 9. (Background information on responses may be found in Blackman and Tukey, ${ }^{1}$ sections 17 and B17, and in Whittlesey, ${ }^{5}$ pp 555-556.)
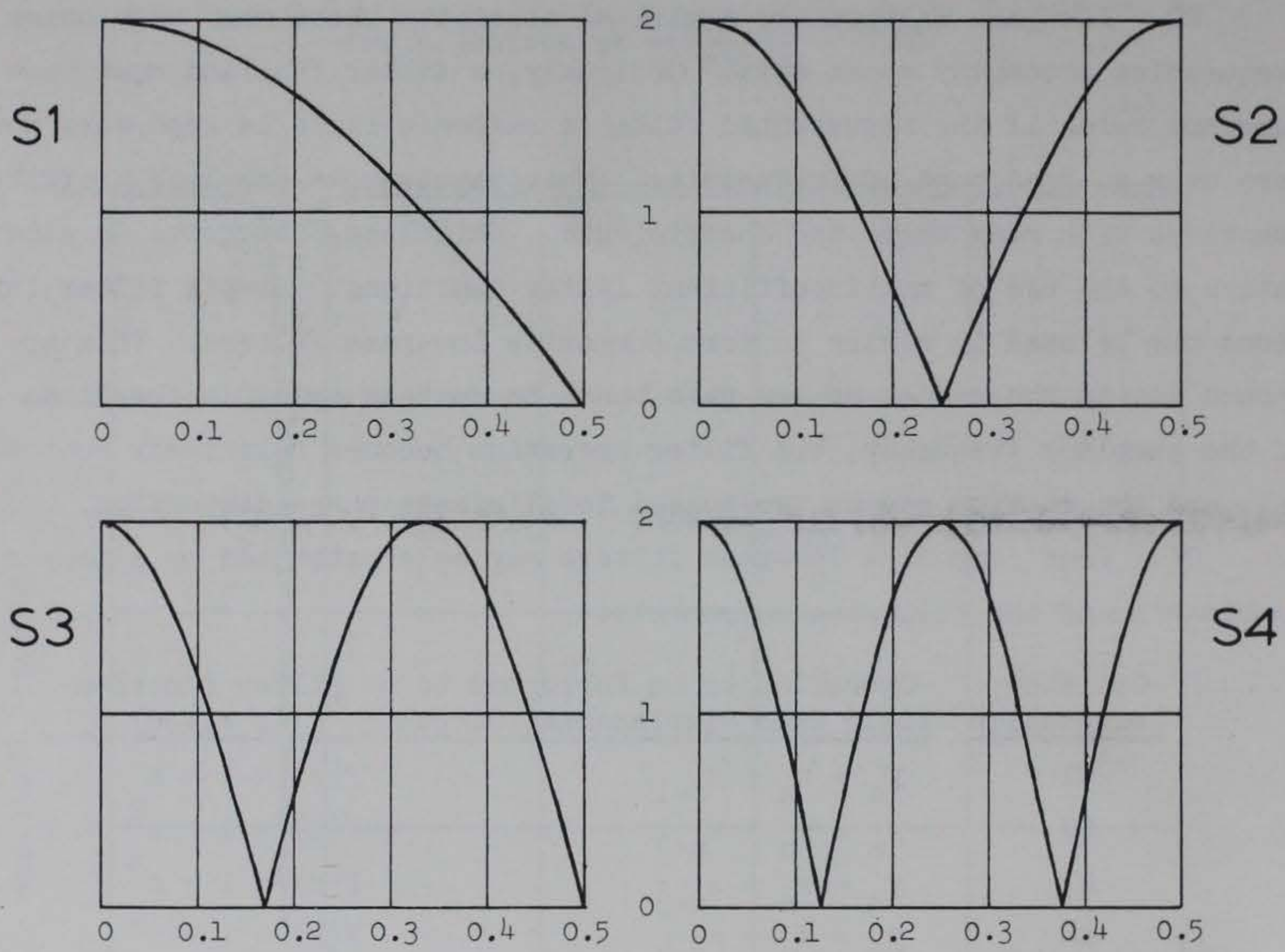

2
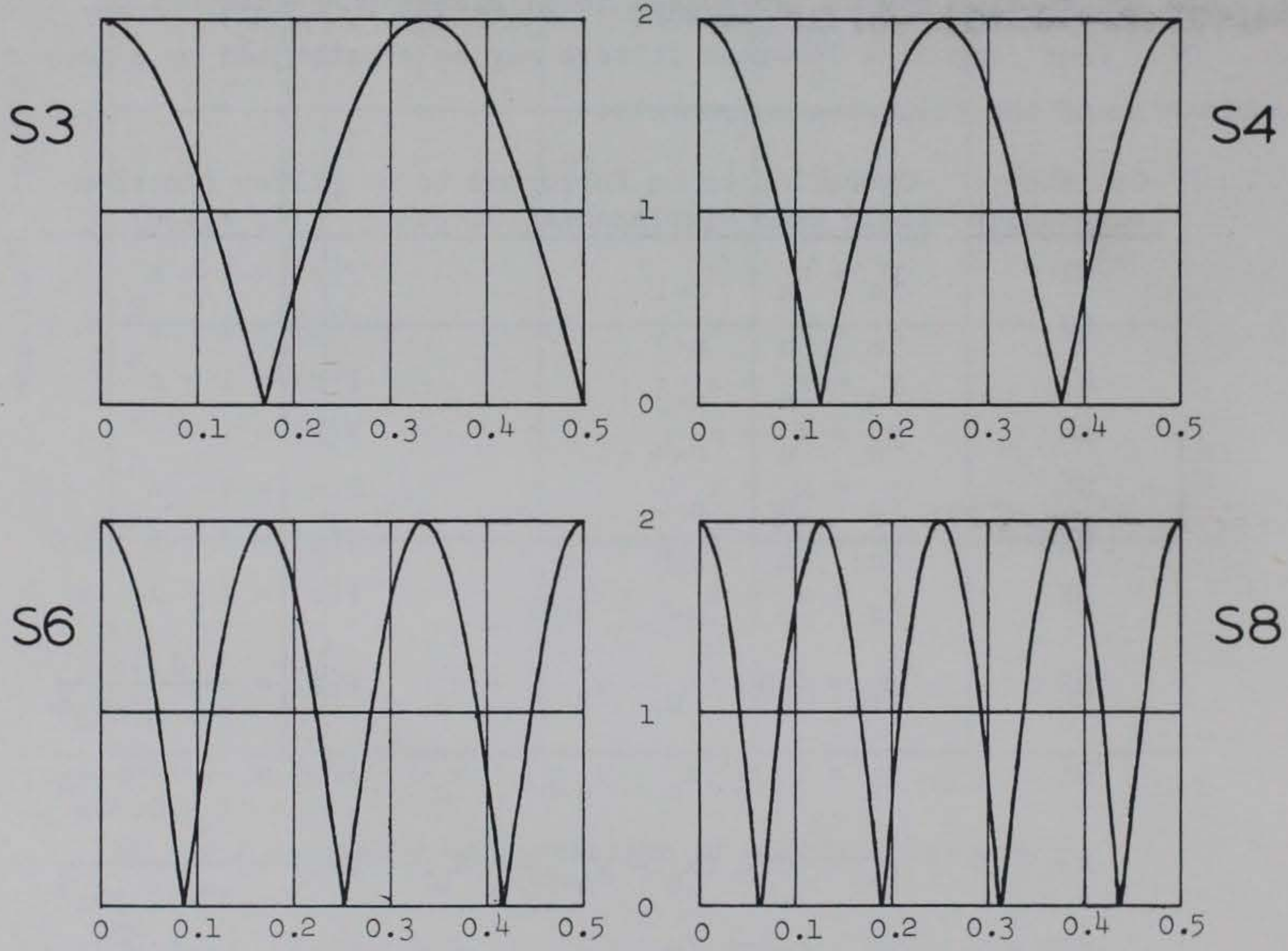

Ordinates: amplitude response. Abscissae: $\frac{f}{f_{s}}$

Fig. 8. Amplitude responses of two-term summing operators (single pass) 

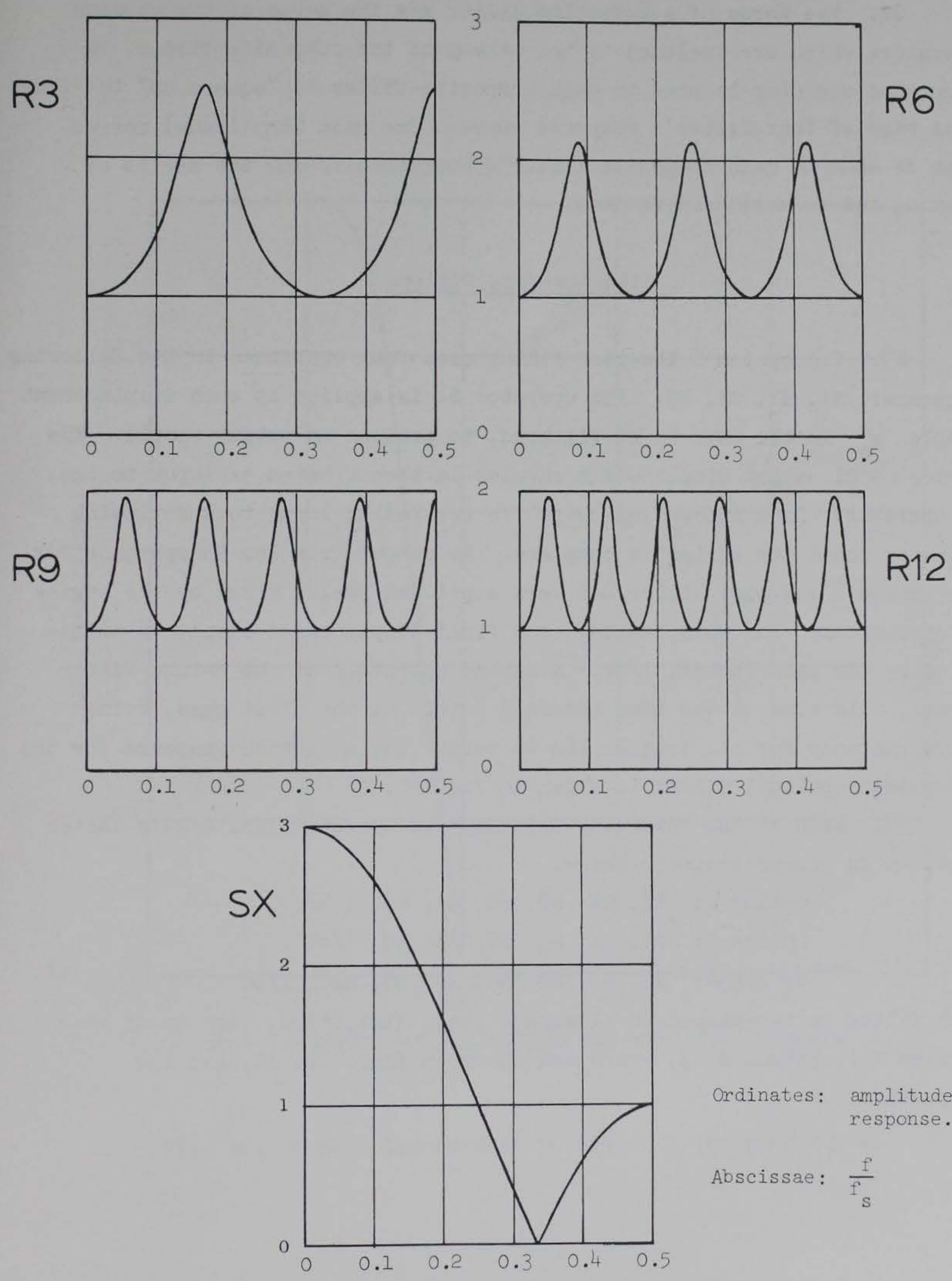

Ordinates: $\begin{aligned} & \text { amplitude } \\ & \text { response. }\end{aligned}$
Abscissae: $\frac{f}{f_{S}}$

Fig. 9. Amplitude response of recursive and threeterm summing operators (single pass) 
22. The zeros of a composite filter are the zeros of the summing operators which are included in the makeup of the composite filter. A recursion operator is used in each composite filter to "square up" the lowpass band of that filter's response curve. One gain (amplitude) correction is made to each composite filter's output following the series of summing and recursion operations.

\section{The Low-Pass Options}

23. The option 1 low-pass filter uses four operators in the following sequence: S1, SX, S2, R3. The operator SI is applied at each displacement sample $x_{n}$ in the wave to be filtered, to produce an output sample. The series of $\mathrm{Sl}$ output displacement samples is then treated as input to the SX operator. Each succeeding output is treated as input to a following operator until the series is complete. An output from the R3 operator for the option 1 low-pass filter has been amplified twelve times by the series of operations. For this reason, each final displacement sample is multiplied by the gain factor, 1/12. A second operation of the entire filter series, this time on the time reversed output of the first pass, brings phase response for all frequencies to zero. The amplitude response for the two-passed option 1 filter is shown in fig. 10.

24. Each of the remaining options uses operators and a gain factor as shown in proper sequence below:

$$
\begin{aligned}
& \text { Option 2: } \mathrm{S} 1, \mathrm{SX}, \mathrm{S} 2, \mathrm{~S} 2, \mathrm{~S} 4, \mathrm{R} 6,1 / 48 \\
& \text { Option 3: } \mathrm{S} 1, \mathrm{SX}, \mathrm{S} 3, \mathrm{~S} 2, \mathrm{~S} 6, \mathrm{R} 9,1 / 48 \\
& \text { Option 4: } \mathrm{S} 1, \mathrm{SX}, \mathrm{S} 2, \mathrm{~S} 4, \mathrm{~S} 2, \mathrm{~S} 8, \mathrm{R} 12,1 / 96
\end{aligned}
$$

Each filter is two-passed to eliminate phase distortion. Amplitude responses for options 2, 3, and 4 are shown in figs. 11, 12, and 13, respectively. 


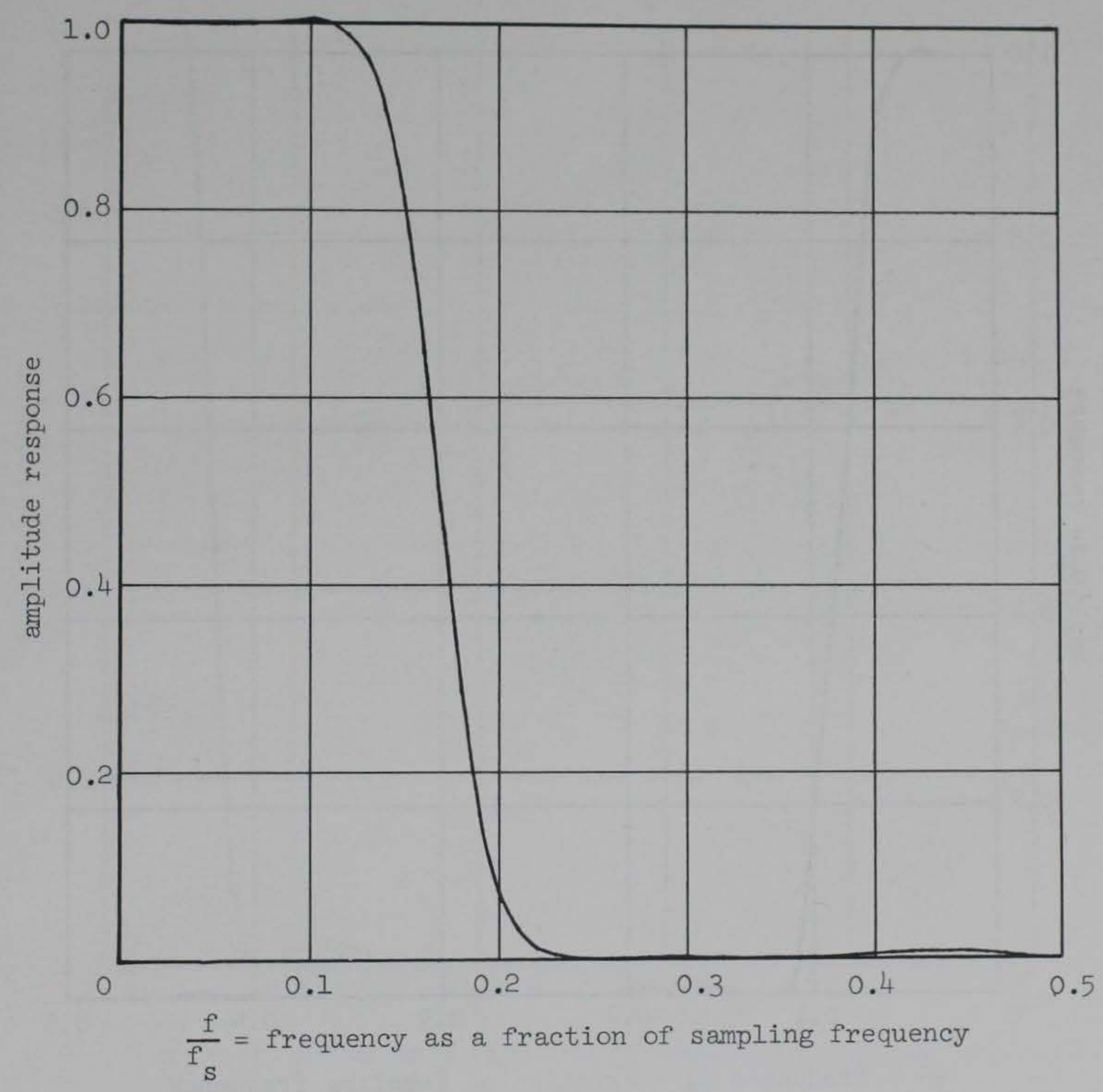

Fig. 10. Amplitude response of option 1 low-pass filter 


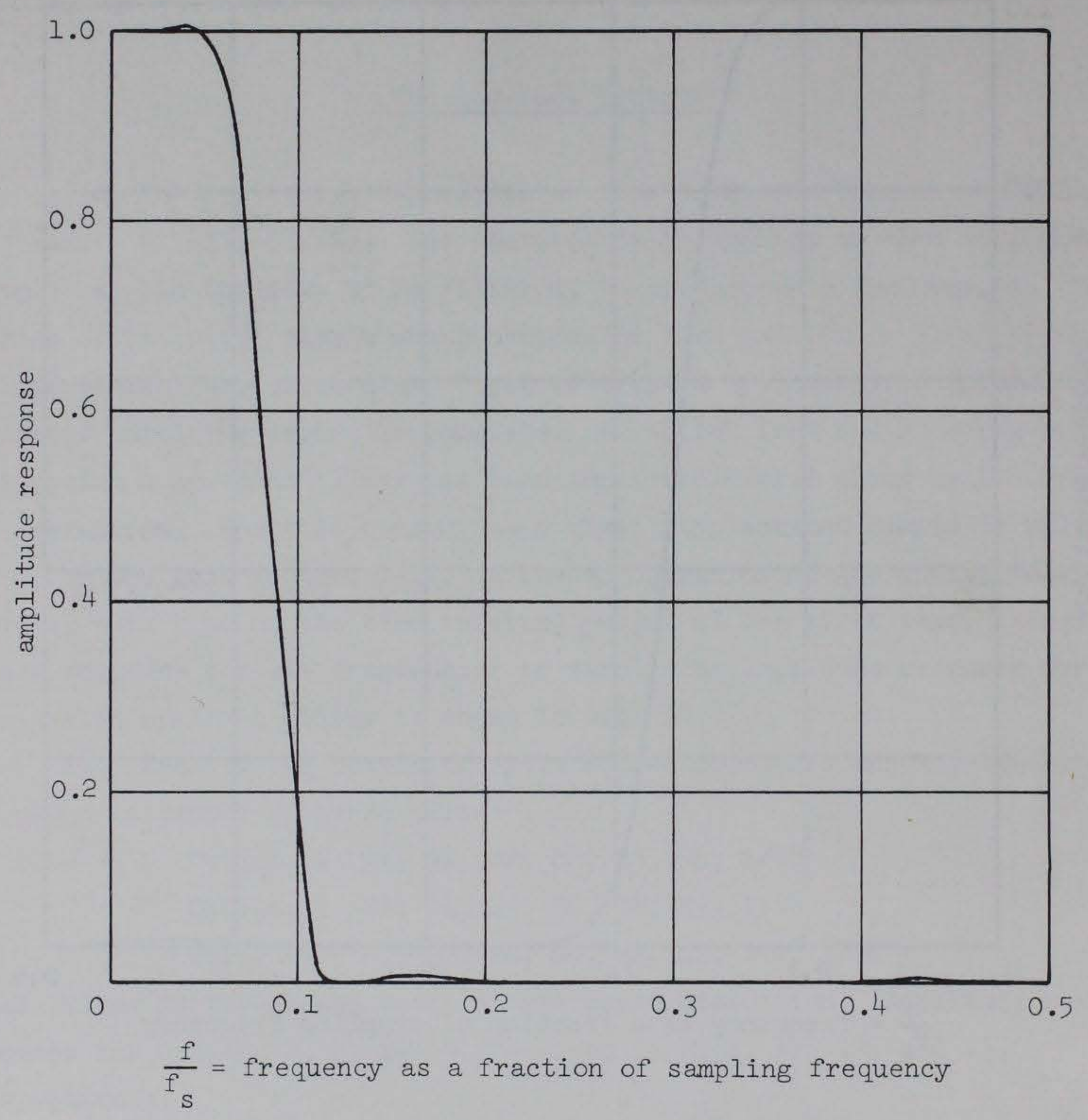

Fig. 11. Amplitude response of option 2 low-pass filter 


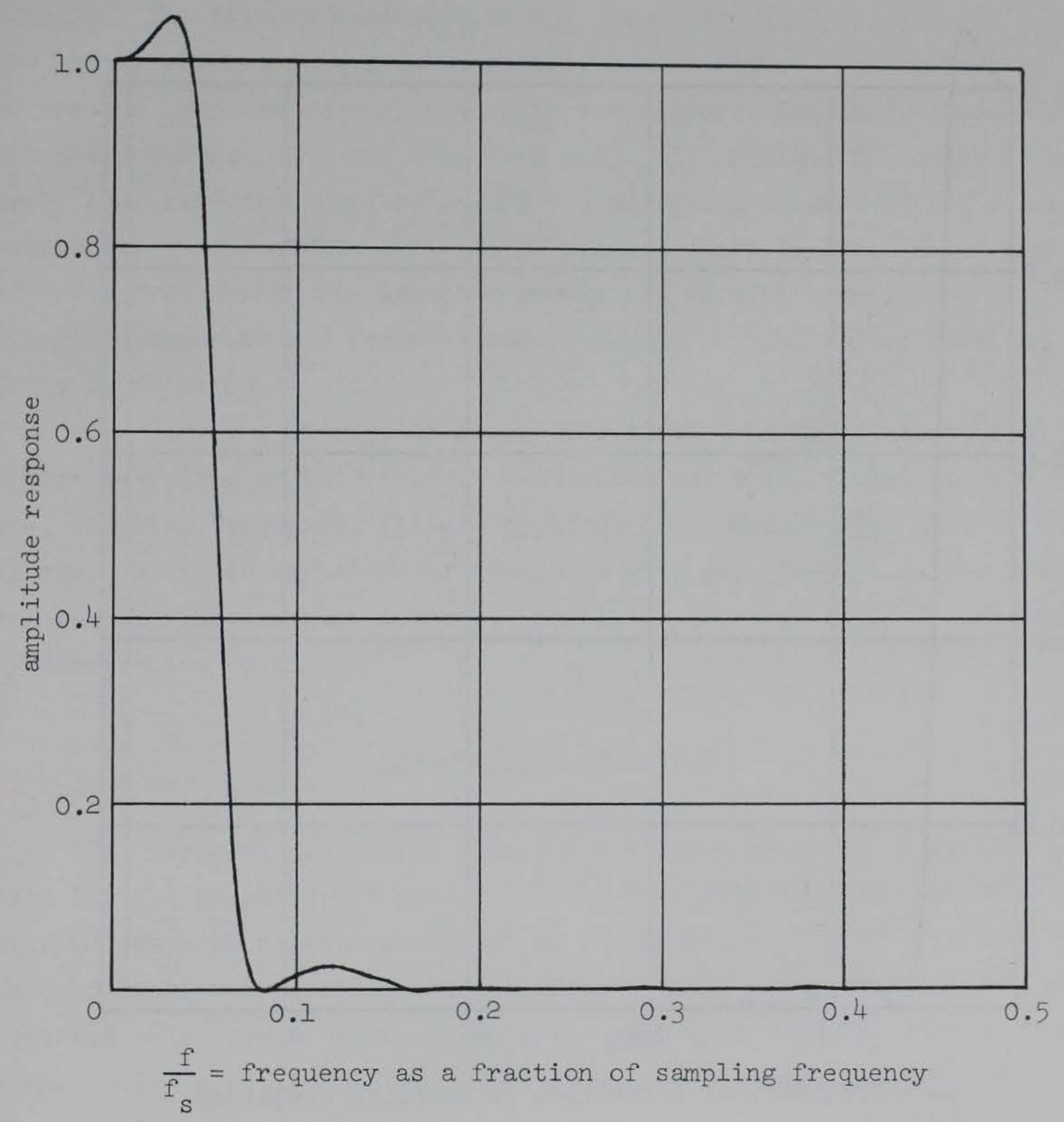

Fig. 12. Amplitude response of option 3 low-pass filter 


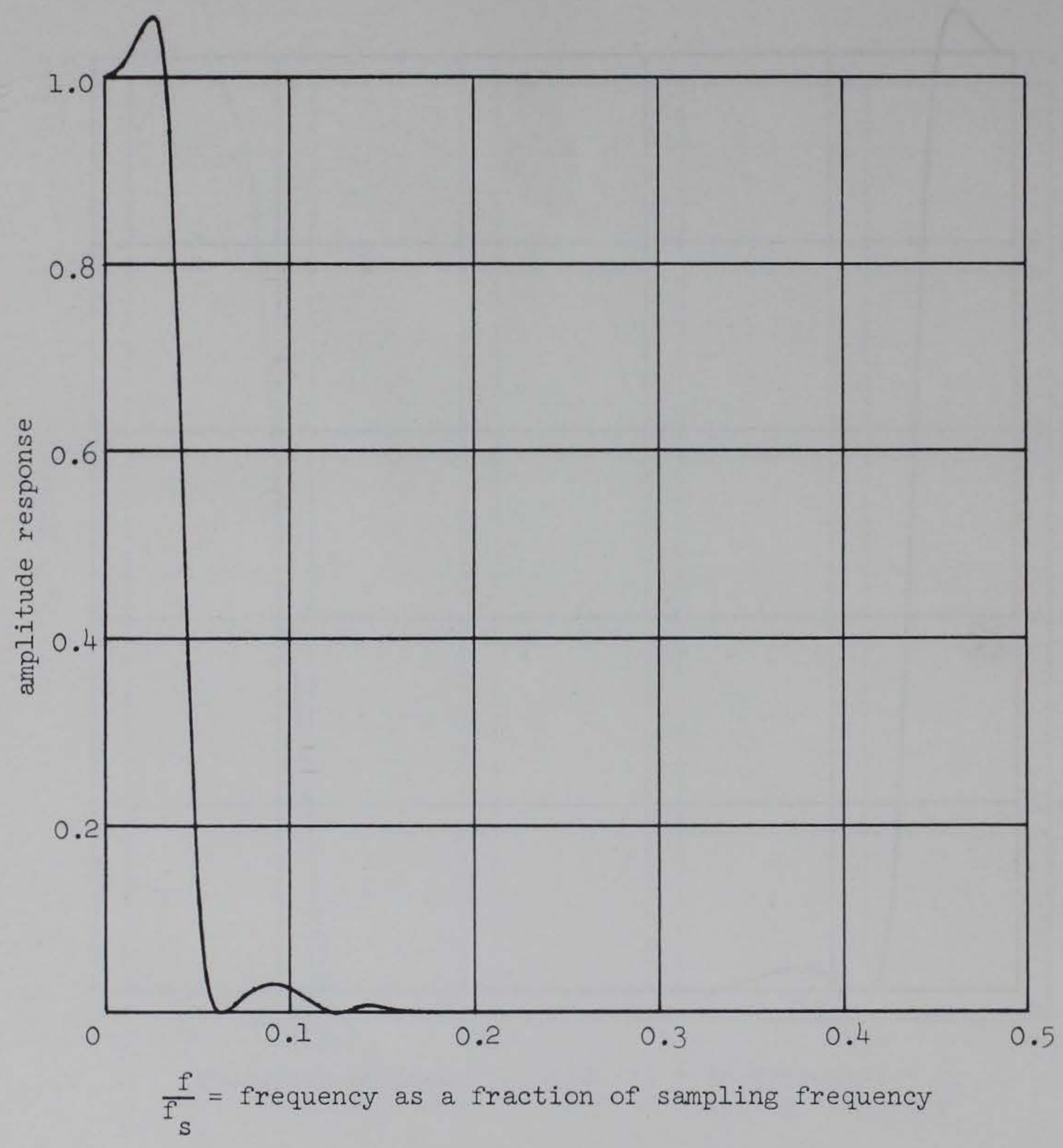

Fig. 13. Amplitude response of option 4 low-pass filter 


\section{General}

25. The filters described in this paper depend for operation upon data inputs which are sampled at equally spaced times. An examination of the various response curves shows that the filters' frequency characteristics are dependent upon sampling frequency. If sampling frequency is relatively low, response curve roll-offs and rejection notches affect fewer frequencies than would be affected if sampling were faster. Where signal and noise frequencies are in close proximity, careful comparisons of processing requirements and response curves should be made before sampling frequency is selected.

26. Plates $1-10$ show examples of digital data improvement using the filters described in this paper. Each plate has been labeled to show gage type, sampling frequency, filters applied, and computer time used in the filters. Where integrations are included with gage data, they are produced from raw or filtered gage outputs. No filter has been applied directly to any integration output.

\section{Random-Spike Rejection}

27. Large single sample spikes affect data plots as shown in plate 1. The computer-set scales for the accelerometer plot create a lowamplitude data presentation because of the spurious spikes' high amplitude. A second problem is seen in the integration to velocity; the spikes have distorted this curve. Plate 2 shows the same data after operation of the random spike rejection filter. Where used, this subroutine precedes frequency filtering operations.

\section{Low-Pass Operations}

28. Plates 2, 5, and 7 show data affected by high noise frequencies, while plates 3,6 , and 8 show the same data after application of 
appropriate low-pass filters. The strain gage data in plates 7 and 8 were sampled very rapidly because it was included on a tape with accelerometer data. Use of the option 4 low-pass filter was necessary in this case. All four low-pass options are included in one NWED subroutine.

\section{Selective Frequency Rejection}

29. The integration to velocity in plate 3 clearly indicates accumulation of area between the accelerometer plot and its zero ordinate--i.e., an offset. This offset, though very small, distorts the integration plots which are intended to represent particle velocity and displacement. Plate 4 shows the same data after application of the zero-frequency rejection filter to the accelerometer output. This filter should be used with care, since surface waves, if present, are likely to fall in the rejection notch. Also, success with zero-frequency rejection depends on signal oscillation which centers approximately on its trace's equilibrium position. Large offsets are handled by making an estimated base-line shift before filtering.

30. The $960-\mathrm{Hz}$ periodic wave on the stress signal in plate 9 has been removed in plate 10 by application of the single-frequency rejection filter. Note retention of the sharp peak at $1.2 \mathrm{msec}$. This characteristic would have been eliminated by low-pass filtering for the $960-\mathrm{Hz}$ noise.

31. The subroutine containing zero- and single-frequency rejection filters is applicable to noise frequencies which are periodic over the time history to be plotted. Its usefulness for particular jobs should be determined from examination of frequency spectra and response curves. Rejection notches may not be overlapped where any frequency between the intended frequencies must be retained at full amplitude.

\section{Exercise of Judgment}

32. Digital filters are quite powerful. Nevertheless, they are like other tools in that they may be misused. Though the filters here discussed are applicable to digitized time histories in general, their blind application to all data will only waste time and money. Professional judgment must guide filter use. 
1. Blackman, R. B. and Tukey, J. W., "The Measurement of Power Spectra, from the Point of View of Communications Engineering," The Bell System Technical Journal, Vol 37, Jan and Mar 1958, pp 185-288, 485-569.

2. LePage, W. R., Complex Variables and the LaPlace Transform for Engineers, McGraw-Hill, New York, 1961.

3. Robinson, E. A. and Treitel, S., "Principles of Digital Filtering," Geophysics, Vol 29, No. 3, June 1964, pp 395-404.

4. Shanks, J. L., "Recursion Filters for Digital Processing," Geophysics, Vol 32, No. 1, Feb 1967, pp 33-51.

5. Whittlesey, J. R. B., "A Rapid Method for Digital Filtering," Communications of the Association for Computing Machinery, Vol 7, No. 9, Sept 1964, pp 552-556. 

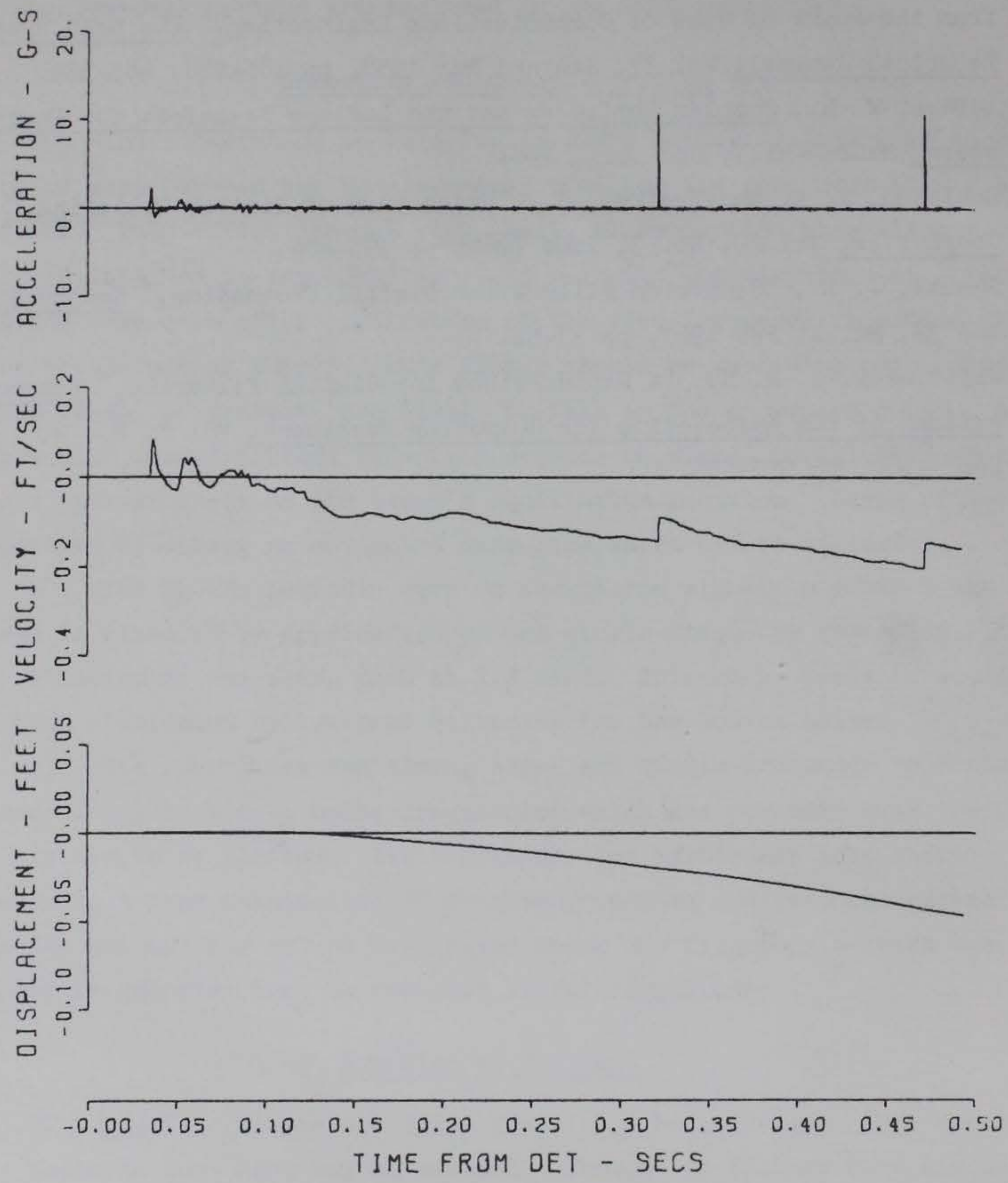

Gage type: Accelerometer Sampling frequency: 6000 samples per second Filters applied: None 


\section{DIODE TUBE \\ $14 C-27-A R$}
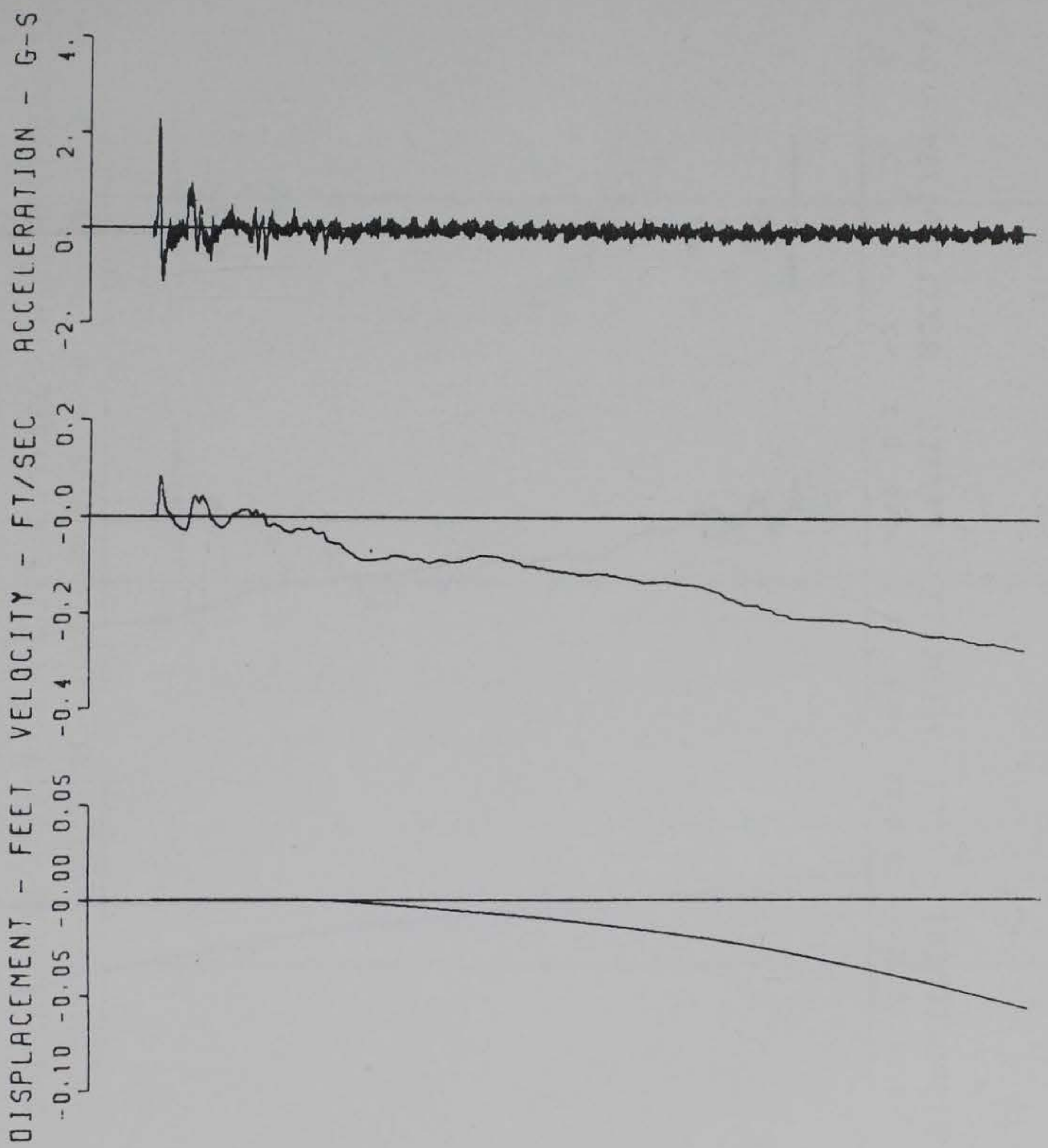

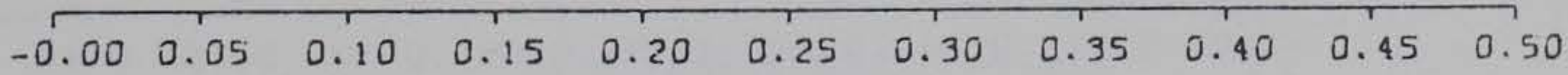
TIME FROM DET - SECS

Gage type:

Sampling frequency: Filter applied: Time in filter:
Accelerometer

6000 samples per second

Random spike rejection

0.03 minute per thousand samples 

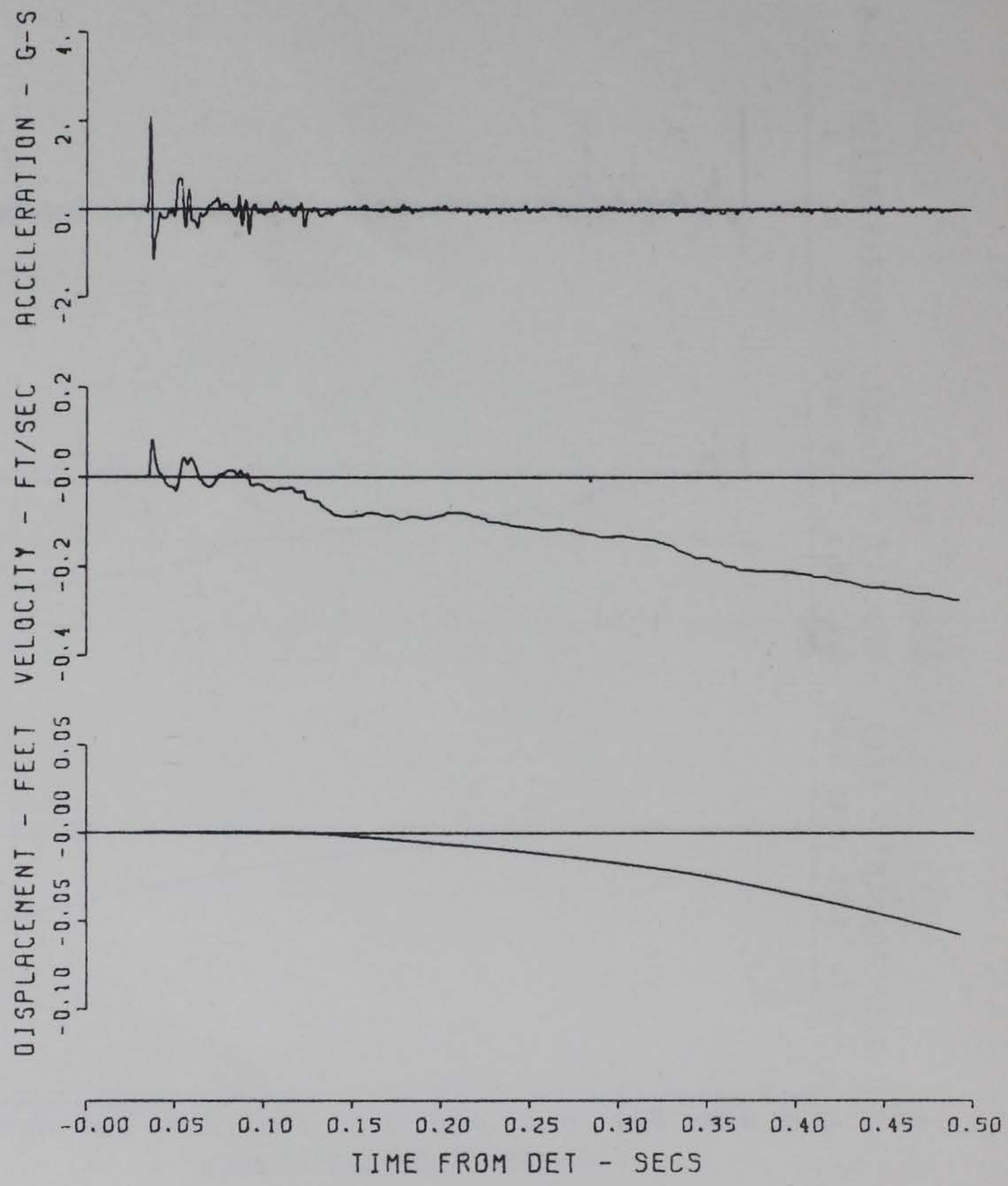

Gage type:

Sampling frequency: Filters applied:

Time in filters:
Accelerometer

6000 samples per second

1. Random spike rejection

2. Option 2 low-pass

0.17 minute per thousand samples 

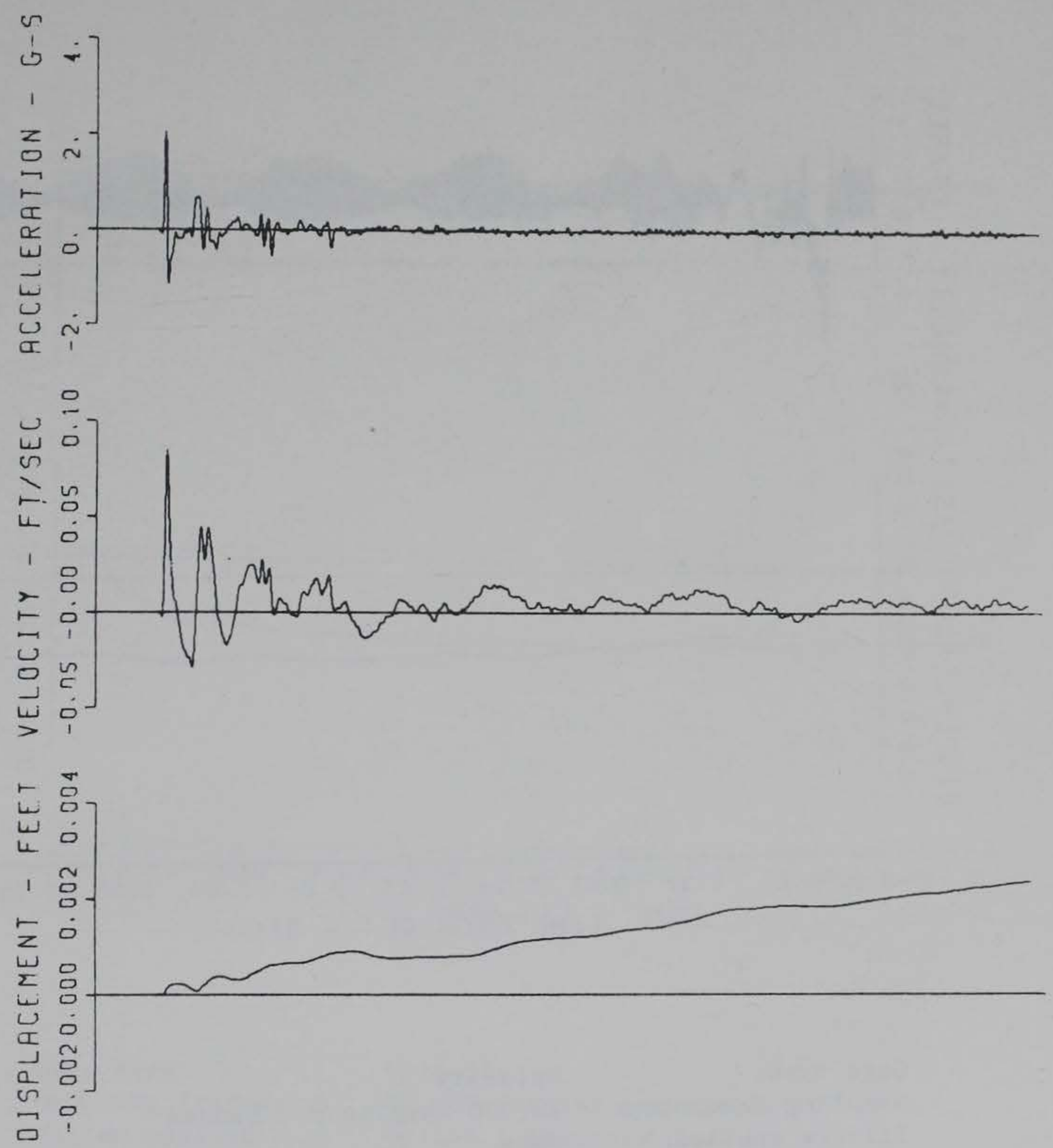

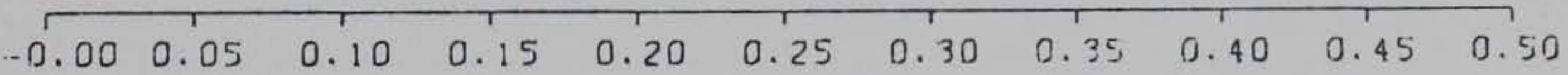
TIME FROM DET - SECS

Gage type:

Sampling frequency: Filters applied:

Time in filters:
Accelerometer 6000 samples per second 1. Random spike rejection

2. Option 2 low-pass

3. Zero frequency rejection

0.28 minute per thousand samples 

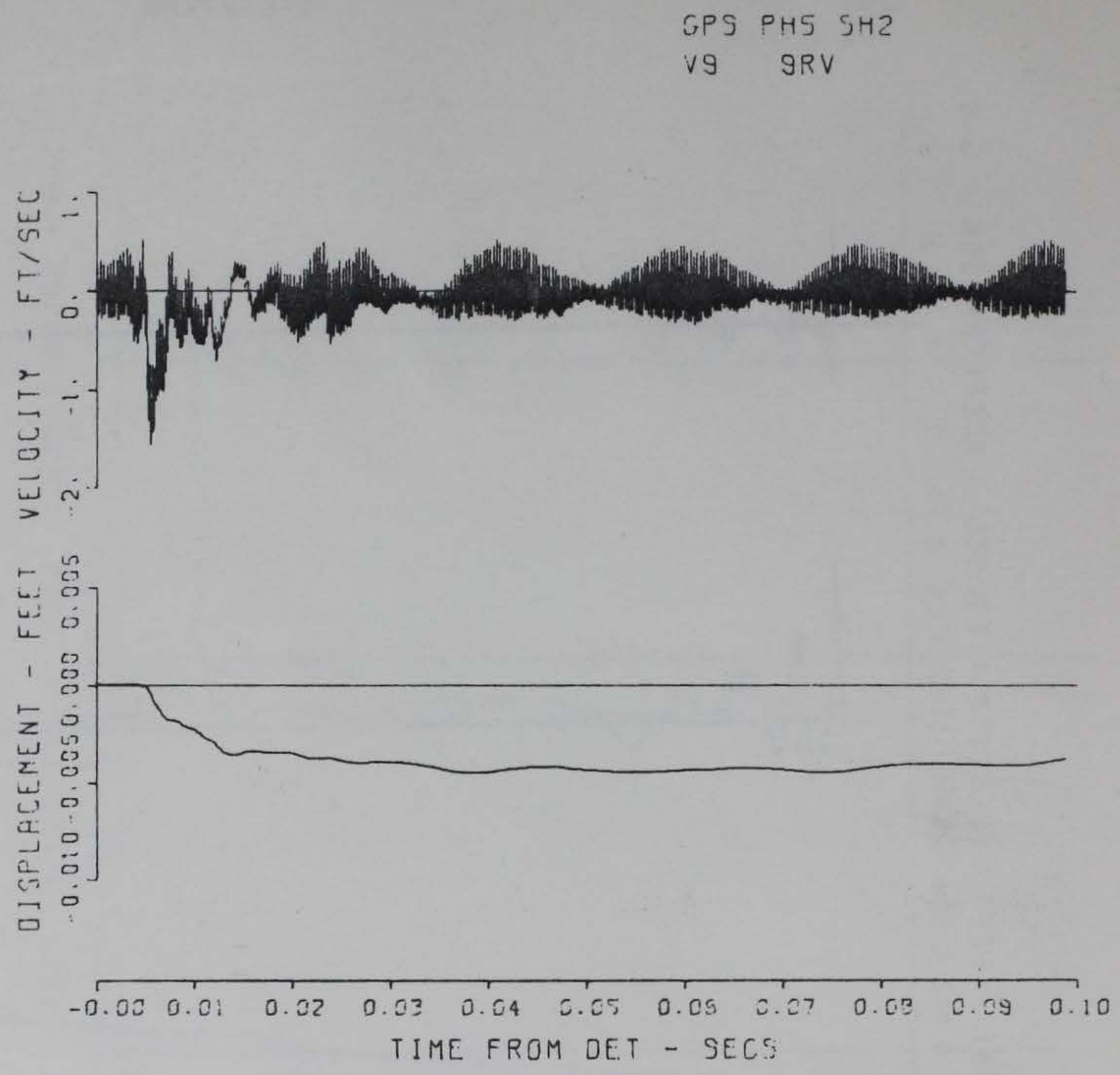

Gage type:

Sampling frequency: Filters applied:
Velocity

12,000 samples per second None 
GPS PHS SHZ

V $9 R V$

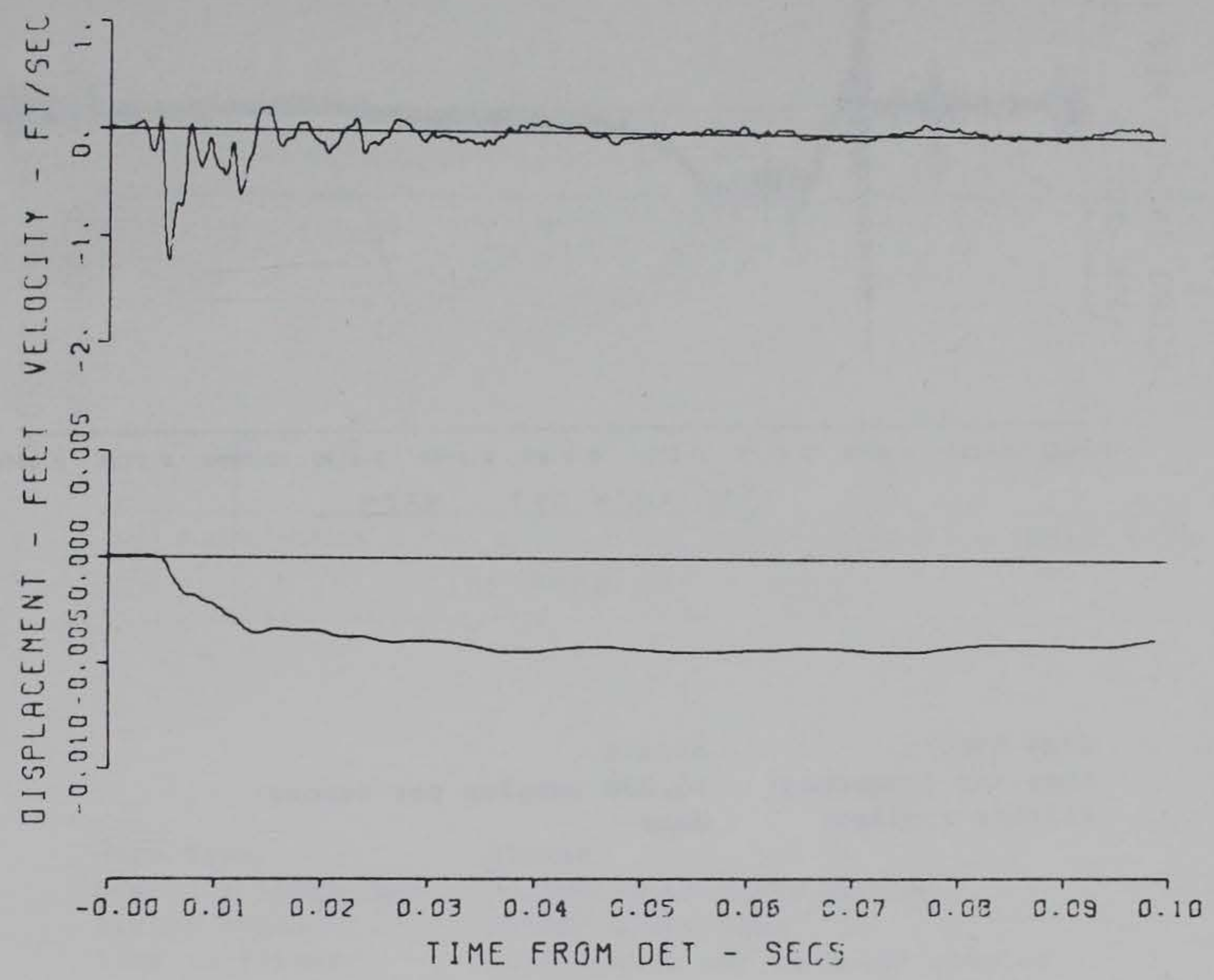

Gage type:

Sampling frequency: Filter applied:

Time in filter:
Velocity

12,000 samples per second

Option 1 low-pass

0.12 minute per thousand samples 

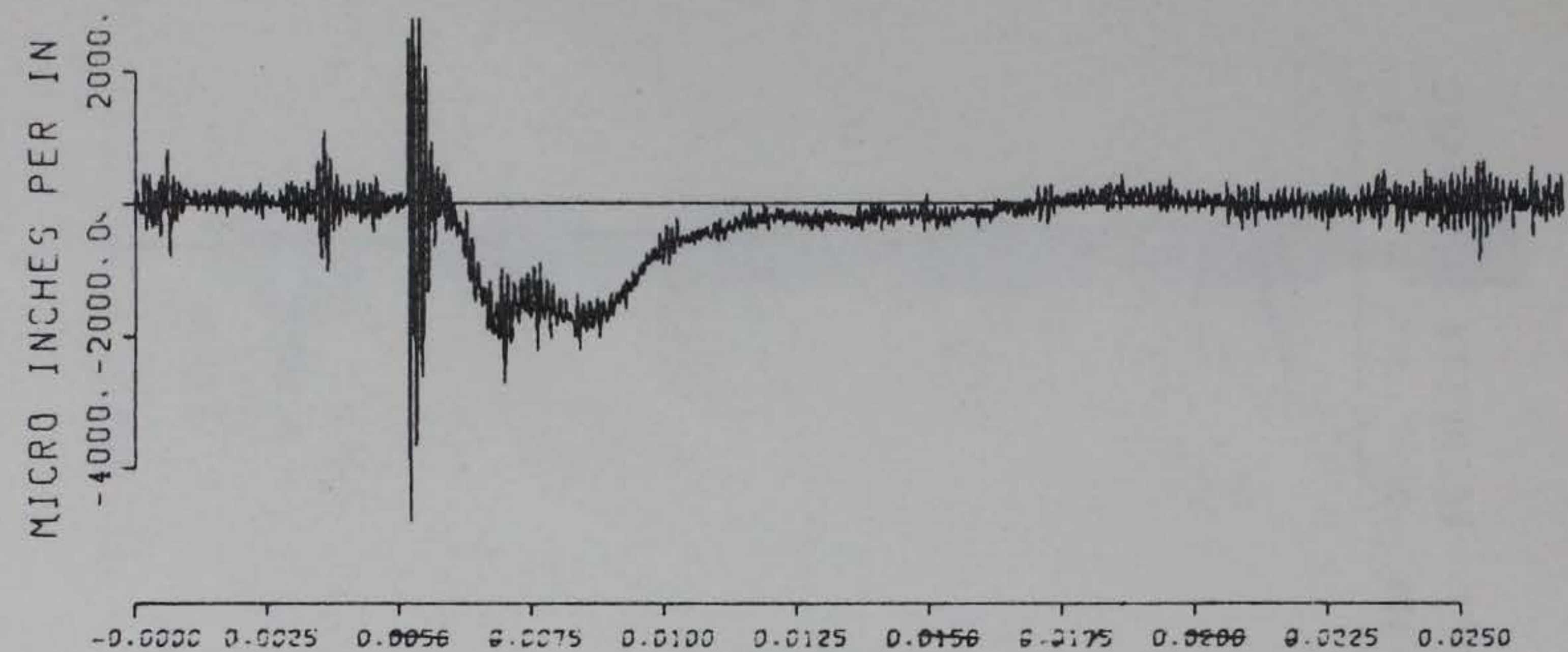

TIME FROM DET - SECS

$\begin{array}{ll}\text { Gage type: } & \text { Strain } \\ \text { Sampling frequency: } & 96,000 \text { samples per second } \\ \text { Filters applied: } & \text { None }\end{array}$


MS-MR OEEP SLFB

554

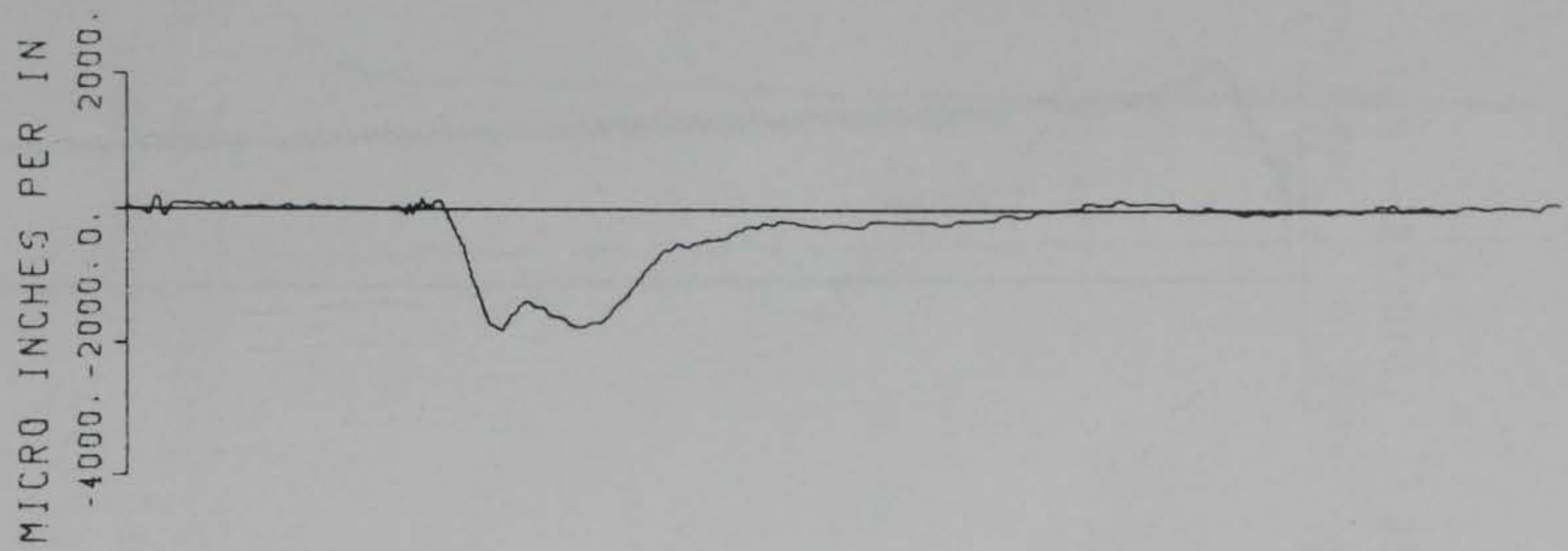

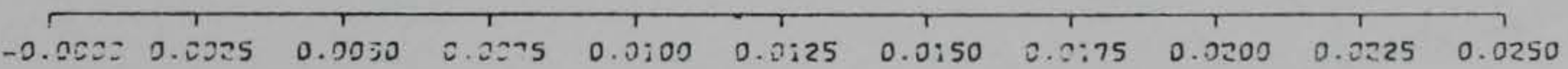

TIME FROM OET - SECS

Gage type:

Sampling frequency:

Filter applied:

Time in filter:
Strain

96,000 samples per second

Option 4 low-pass

0.18 minute per thousand samples 


\section{SPS PH4A SH1 \\ E1 OCV}
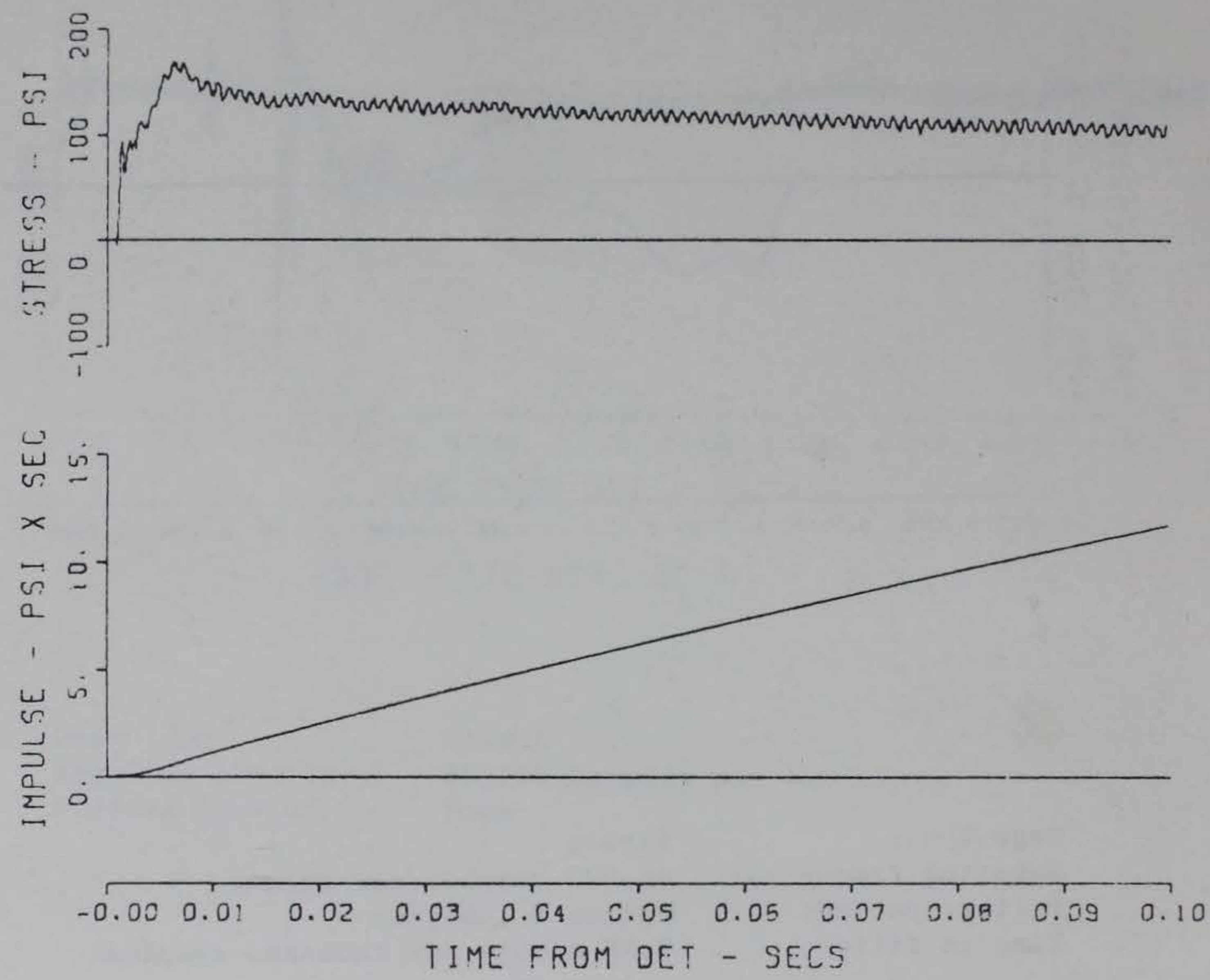

$\begin{array}{ll}\text { Gage type: } & \text { Stress } \\ \text { Sampling frequency: } & 12,000 \text { samples per second } \\ \text { Filters applied: } & \text { None }\end{array}$




\section{GPS PHAF SHI \\ E1 OCV}
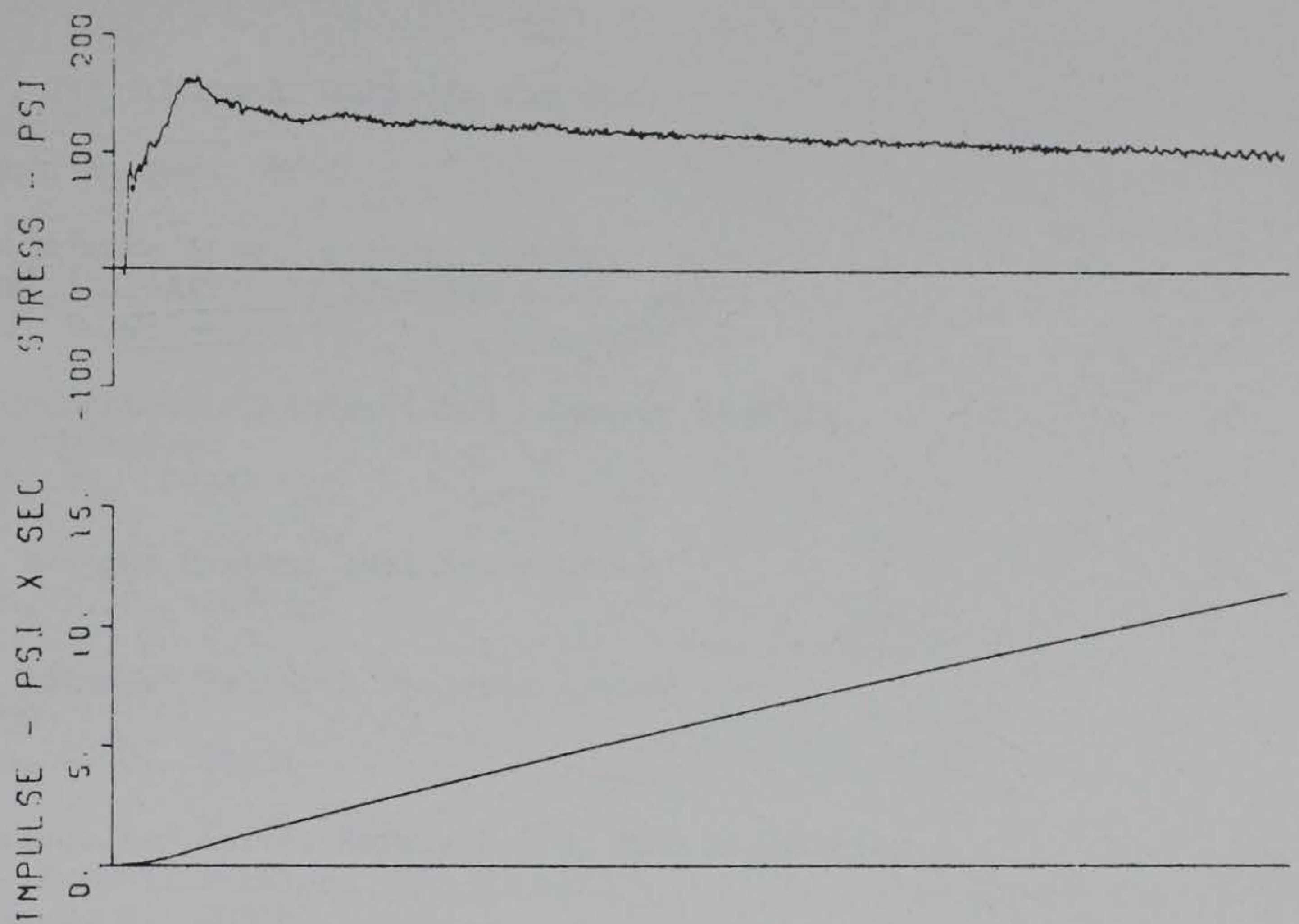

\begin{tabular}{|c|c|c|c|c|c|c|c|c|c|c|}
\hline-0.00 & 0. 0: & 0.02 & C. 03 & 0.04 & 0.05 & 0.05 & 0.07 & 0.03 & 0.09 & 0.10 \\
\hline
\end{tabular}

Gage type:

Sampling frequency: Filter applied: Time in filter:

\section{Stress}

12,000 samples per second

Single frequency rejection ( $960 \mathrm{hz})$

0.25 minute per thousand samples 
Unclassified

Security Classification

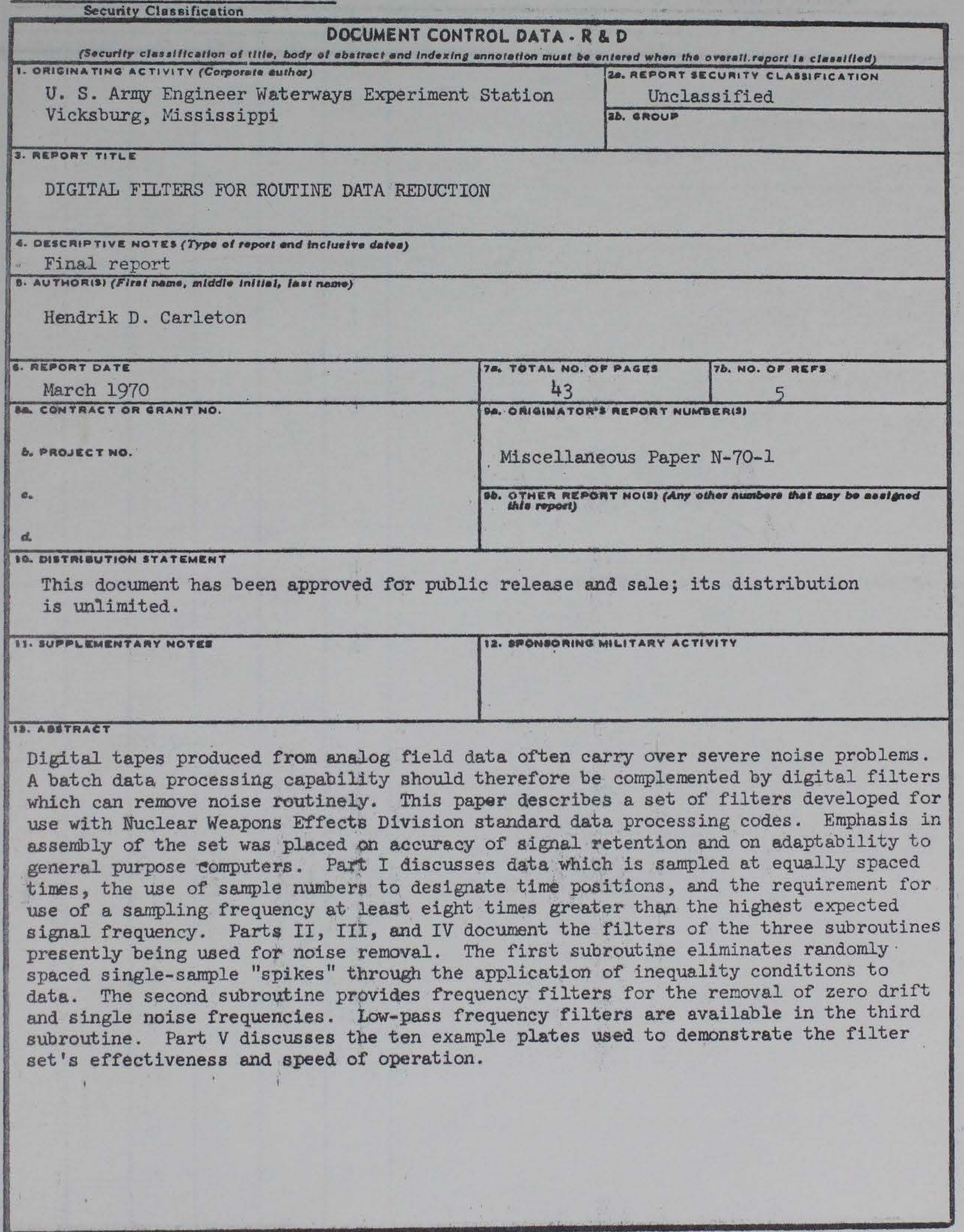




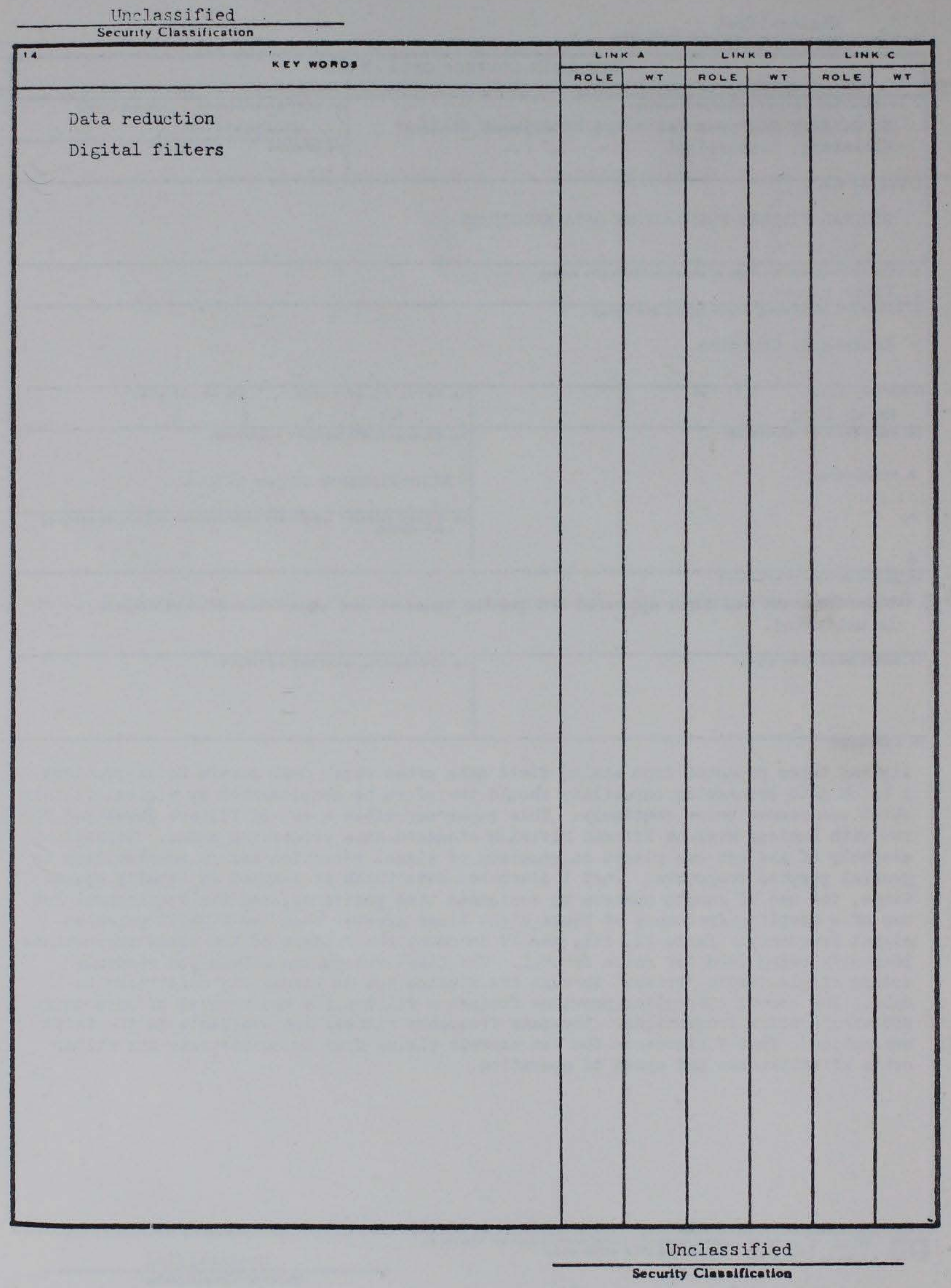

